\title{
Amyloid Precursor Protein (APP) controls excitatory/inhibitory synaptic inputs by regulating the transcriptional activator Neuronal PAS Domain Protein 4 (NPAS4)
}

5 Rémi Opsomer ${ }^{1}$, Sabrina Contino ${ }^{1 \dagger}$, Florian Perrin ${ }^{1,2 \dagger}$, Bernadette Tasiaux ${ }^{1}$, Pierre Doyen ${ }^{3}$,

6 Maxime Vergouts ${ }^{3}$, Céline Vrancx ${ }^{1}$, Anna Doshina ${ }^{1}$, Nathalie Pierrot ${ }^{1}$, Jean-Noël Octave ${ }^{1}$, Serena

7 Stanga $^{1}$, Pascal Kienlen-Campard ${ }^{1 *}$.

$8{ }^{1}$ CEMO-Alzheimer Dementia group, Institute of Neuroscience, Université catholique de Louvain,

9 Brussels, Belgium.

102 de Duve Institute, Ludwig Institute for Cancer Research and Université catholique de Louvain,

11 Brussels, Belgium.

$12{ }^{3}$ CEMO-Laboratory of Neuropharmacology, Institute of Neuroscience, Université catholique de

13 Louvain, Brussels, Belgium.

14 †These authors have contributed equally to this work.

15 *Correspondence:

16 Pascal Kienlen-Campard

17 Institute of Neuroscience

18 IONS-CEMO, Avenue Mounier 53 bte B1.53.02

19 B-1200 Brussels

20 Belgium

21 Phone: +3227649335

22 Fax: +3227645460

23 Email: pascal.kienlen-campard@uclouvain.be

24

\section{Running title}

APP-dependent NPAS4 expression and GABA production.

\section{Keywords}

Alzheimer's disease; APP family proteins; Neuronal differentiation; Transcriptome analysis; 


\section{Abstract}

34 Sequential proteolysis of the amyloid precursor protein (APP) and amyloid- $\beta$ peptide (A $\beta$ ) release

35 is an upstream event in Alzheimer's disease (AD) pathogenesis. The function of APP in neuronal

36 physiology is still, however, poorly understood. Along with its paralog APP-like Proteins 1 and 2

37 (APLP1-2), APP is involved in neurite formation and synaptic function by mechanisms that are

38 not elucidated. APP is a single-pass transmembrane protein expressed at high levels in the brain

39 that resembles a cell adhesion molecule or a membrane receptor, suggesting that its function relies

40 on cell interaction processes and/or activation of intracellular pathways of signal transduction.

41 Along this line, the APP intracellular domain (AICD) was reported to act as a transcriptional factor

42 for targeted gene activation that mediates physiological APP functions. Here, we used an unbiased

43 transcriptome-based approach to identify the genes transcriptionally regulated by APP in the rodent

44 embryonic cortex and upon maturation of primary cortical neurons. The transcriptome analysis did

45 not detect any significant differences in expression of previously proposed AICD target genes. The

46 overall transcriptional changes were subtle, but we found that genes clustered in neuronal-activity

47 dependent pathways are dysregulated in the absence of APP. Among these genes, we found the

48 activity-dependent Neuronal PAS domain protein 4 (NPAS4) Immediate Early Gene to be

49 downregulated in the absence of APP. Down-regulation of NPAS4 in APP knock-out (KO) neurons

50 is not related to AICD but to the APP ectodomain. We studied the effect of APP deficiency on

51 GABAergic and glutamatergic transmission, and found an increased production of the inhibitory

52 neurotransmitter GABA in APP KO neurons, along with a reduced expression of the GABA(A)

53 receptors alpha1, suggesting an impaired GABAergic neurotransmission in the absence of APP.

54 CRISPR-Cas-mediated silencing of NPAS4 in neurons led to similar observations. Altogether, our

55 results point out a new role for APP in the regulation of excitatory/inhibitory neurotransmission

56 through the regulation of the activity-dependent NPAS4 gene. 


\section{Introduction}

58 The Amyloid Precursor Protein (APP) has been extensively studied as the precursor of the amyloid-

$59 \beta$ peptide $(A \beta)$, the major component of the senile plaques which are a typical hallmark of

60 Alzheimer's disease (AD). Still, the physiological functions of APP per se have been largely

61 overlooked and remain a matter of controversy. Understanding the physiological function of APP

62 and how its deregulation would contribute to $\mathrm{AD}$ pathogenesis is thus of prime interest.

63 APP is a type 1 transmembrane protein that belongs to the APP-like protein family (APLP1 and 64 APLP2, referred to as APLPs), which are present in most of the species, excepted in yeast, prokaryotes and plants. The APLP family has been generated by several duplications and contraction events during evolution. The specific physiological role and/or redundant functions assigned to each member are yet not clearly defined (for a review see Shariati and De Strooper, 2013). APP-/- mice show a subtle phenotype, with reduced body and brain weight, reduced locomotor activity, gliosis, mild axonal growth/white matter defects and altered long-term potentiation responses (Guo et al., 2012; Muller et al., 2012; Muller and Zheng, 2012). In this broad (and complex) picture, growing evidence indicate that APP controls neuronal proliferation, differentiation (Freude et al., 2011; Hu et al., 2013) and migration during embryogenesis (YoungPearse et al., 2007). APP contributes to the establishment of a functional neuronal network by promoting neurite outgrowth (Hoe et al., 2009b). Additionally, APP was reported to control synaptic formation and activity (Priller et al., 2006; Santos et al., 2009; Lee et al., 2010; Pierrot et al., 2013; Klevanski et al., 2015; Zou et al., 2016) in the central nervous system (CNS) and at the neuromuscular junction (Stanga et al., 2016). APP directly modulates the excitatory neurotransmission by interacting with AMPA (Lee et al., 2010) or NMDA (Cousins et al., 2009; Hoe et al., 2009a) receptors. APP was also described to play an important role in GABAergic inhibitory neurotransmission. APP deficiency reduces paired pulse depression (PPD) in mice (Seabrook et al., 1999) and affects GABA receptors expressions (Fitzjohn et al., 2000; Chen et al., 2017), while APP overexpression induces hyperexcitability due to GABAergic neurotransmission failure (Born et al., 2014). Recently, APP was associated with the GABA excitatory/inhibitory shift occurring in embryonic neurons (Doshina et al., 2017). APP appears therefore to have a direct role in the fine-tuning of excitatory and inhibitory neurotransmission, a process that seems to be also critical in $\mathrm{AD}$ pathogenesis.

87 Tuning inhibitory/excitatory neurotransmission is very important for neuronal plasticity and memory formation. This is regulated by a specific subset of genes induced by neuronal activity, belonging to the Immediate Early Genes (IEGs) family, which control the mechanisms that "reshape" synaptic inputs on neurons (West and Greenberg, 2011). IEGs expression is instrumental to neuronal plasticity and memory formation (Alberini, 2009; Loebrich and Nedivi, 2009; Leslie and Nedivi, 2011). Among these IEGs, NPAS4 is specifically involved in a transcriptional program that regulates neuronal firing responses to excitatory transmission by enhancing inhibition (Lin et al., 2008), therefore keeping neuronal firing in response to stimuli within normal levels (Spiegel et 
96 excitation onto the same neurons (Spiegel et al., 2014). NPAS4 is therefore a key player in the 97 maintenance of excitatory/inhibitory balance in neuronal network.

98 The precise mechanisms underlying APP synaptic functions are still elusive. One could suspect APP to regulate the expression of genes involved in synaptic activity, or to shape the structure of 100 the synapse. APP was shown to control gene expression through its intracellular domain called 101 AICD. An increasing list of AICD candidate genes has emerged from various models (reviewed in 102 Pardossi-Piquard and Checler, 2012). Some of these candidate genes failed to be confirmed by 103 transcription analysis in APP-deficient cell lines (Hebert et al., 2006; Waldron et al., 2008), and 104 APP was also reported to regulate gene transcription independently of AICD release (Hicks et al., 105 2013; Pierrot et al., 2013). It is so far impossible to clearly define (i) the precise identity of APP 106 target genes in neurons (ii) how these APP target genes relate to APP neuronal function (iii) the 107 mechanism involved in APP-dependent in gene transcription.

108 In the present study, we first aimed at identifying genes transcriptionally regulated by APP in 109 primary neurons. To that end, we performed a non-biased transcriptome analysis of APP $+/+$ and 110 APP-/- primary cortical neurons at different stage of differentiation. In-depth transcriptome 111 analysis revealed that the absence of APP induced only subtle changes in global gene expression. 112 The hitherto described AICD target genes were not significantly up-or down-regulated in our 113 model. A more detailed analysis indicates that expression of genes clustered in specific neuronal 114 pathways was affected by the absence of APP. In particular, the transcription of the activity115 dependent transcription factor Npas4 gene was down-regulated in the absence of APP after 7 116 days of culture. Interestingly, we observed that the amount of the inhibitory neurotransmitter $\gamma$ 117 aminobutyric acid (GABA) and the expression of glutamate decarboxylase 65 (GAD65), the 118 enzyme that catalyzes the decarboxylation of glutamate to GABA, were increased in APP-/119 neurons, suggesting that the inhibitory inputs in synaptic transmission are increased in APP KO 120 neurons. Direct down-regulation of Npas4 by CRISPR-Cas9 editing in neurons mimicked the 121 increase in GAD65 and GABA release observed in APP-/- cultures. Altogether, our data give a 122 new in APP-dependent neuronal activity, supporting that APP tunes the excitatory/inhibitory 123 transmission in neuronal networks. 


\section{Materials and Methods}

\section{Antibodies, chemicals and reagents}

126 All media and reagents used for cell cultures were purchased from Thermo Fisher Scientific 127 (Waltham, MA); fetal bovine serum was purchased from Biowest (Nuaillé, France). Analytical 128 grade solvents and salts were purchased from Sigma-Aldrich (St-Louis, MO). N-[N-(3,5129 Difluorophenacetyl)-L-alanyl]-S-phenylglycine t-butyl ester (DAPT), sAPP $\alpha$ (S9564) and DAPI 130 (D9542) were from Sigma-Aldrich (St-Louis, MO, USA). Triton-X100 was purchased from Merck 131 (Darmstadt, Germany) and TriPure Isolation Reagent from Roche (Basel, Switzerland). Microarray 132 analysis kits were from Affymetrix (Santa Clara, CA, USA). All reagents for RNA processing or 133 cDNA synthesis were purchased from Bio-Rad (Hercules, CA). Primers were purchased from 134 Sigma-Aldrich (St Louis, MO, USA). Proteins were quantified with BCA Protein Assay kit 135 (Thermo Fisher Scientific, Waltham, MA). NuPAGE® reagents were from Invitrogen (Carlsbad, $136 \mathrm{CA}$ ). PVDF and nitrocellulose membranes were from Merck Millipore (Billerica, MA,) or 137 Amersham $^{\mathrm{TM}}$ (Little Chalfont, UK). Nonfat dry milk was from Merck (Darmstadt, Germany). 138 Western Lighting® Plus-ECL reagents were from PerkinElmer (Waltham, MA) and Fluoprep 139 mounting medium was from bioMérieux (Marcy l'Etoile, France). Lentivirus were prepared with 140 Acrodisc ${ }^{\circledR}$ 0,45 $\mu \mathrm{m}$ filters (Pall, NYC, USA) and LentiX ${ }^{\mathrm{TM}}$ Concentrator reagent (Clontech, 141 Mountain View, CA).The following antibodies were used: APP NT (22C11, MAB348, Merck 142 Millipore, Billerica, MA), anti-human APP (WO2, MABN10, Merck Millipore, Billerica, MA), 143 anti-APP CT (Y188, Abcam, Cambridge, UK), anti-APLP1 (Cat. No. 171615, Calbiochem EMD 144 Biosciences - Merck, Darmstadt, Germany), anti-APLP2 (Cat. No. 171616, Calbiochem EMD 145 Biosciences - Merck, Darmstadt, Germany), anti-GAPDH (14C10, Cell Signaling, Danvers, MA, 146 USA), anti-MAP2 (M4403, Sigma-Aldrich St Louis, MO), anti-GAD65 (D5G2, Cell Signaling), 147 anti-mouse IgG, HRP Whole antibody (NA931-1ML, Amersham, Little Chalfont, UK), anti-rabbit 148 IgG, HRP Whole antibody (NA934-1ML, Amersham, Little Chalfont, UK), goat anti-mouse Alexa 149 Fluor ${ }^{\circledR}-488$, goat anti-mouse Alexa Fluor®-568, goat anti-rabbit Alexa Fluor ${ }^{\circledR} 647$ and DAPI 150 were purchased from ThermoFisher Scientific (Waltham, MA, USA). Glutamate assay kit was 151 from Abcam (Cambridge, UK) and $\gamma$-aminobutyric acid (GABA) ELISA was purchased from 152 Cloud-Clone Corporation. $70 \mu \mathrm{m}$ Falcon ${ }^{\mathrm{TM}}$ Cell Stainers were from ThermoFisher Scientific 153 (Waltham, MA).

\section{Animal models}

$155 \mathrm{APP}+/+$ and APP-/- mice were obtained from the Jackson Laboratory (Bar, Harbor, ME, USA) as $156 \mathrm{C} 57 \mathrm{Bl6} / \mathrm{J}$ and backcrossed for $>6$ generations in CD1 genetic background. Animals were housed 157 on a $12 \mathrm{~h}$ light/dark cycle in standard animal care facility with access to food and water ad libidum. 158 Heterozygous animals (APP+/-) were bred and crossed to obtain embryos from the three different 159 genotypes (APP+/+, APP+/- and APP-/-) in the same litter. All experiments were performed in 160 compliance with protocols approved by the UClouvain Ethical Committee for Animal Welfare 161 (code number 2016/UCL/MD/015). 
163 Primary cultures of cortical neurons were prepared from E18 mouse embryos as previously 164 described (Pierrot et al., 2013). Briefly, cortices were dissected and dissociated in HBSS without 165 calcium and magnesium and the mixture was centrifuged on Fetal Bovine Serum (FBS) for 10 min 166 at $1000 \mathrm{xg}$ to pellet cells. Cells were plated at 200.000 cells $/ \mathrm{cm}^{2}$ in culture dishes pre-treated with $16710 \mu \mathrm{g} / \mathrm{ml}$ of poly-L-lysine in phosphate buffered saline (PBS) and cultured for 3 to 14 days in vitro 168 in Neurobasal ${ }^{\circledR}$ medium enriched with $2 \% \mathrm{v} / \mathrm{v}$ B-27® supplement medium and $1 \mathrm{mM}$ L-glutamine 169 at $37^{\circ} \mathrm{C}, 5 \% \mathrm{CO}_{2}$ and humidified atmosphere. Half of the medium was renewed every 2-3 days.

170 After 6 days (DIV6), neurons were treated for $16 \mathrm{~h}$ with $1 \mu \mathrm{M}$ of $\mathrm{N}$-[N-(3,5-Difluorophenacetyl)-L171 alanyl]-S-phenylglycine t-butyl ester (DAPT), a $\gamma$-secretase inhibitor (Dovey et al., 2001) or with $17220 \mathrm{nM}$ of soluble APP alpha in Neurobasal® Medium

\section{Primary astrocytes culture and treatments}

174 Cortices from rat pups were collected at postnatal day 2 and mechanically dissociated. Astrocytes 175 were isolated using a 30\% Percoll gradient and seeded into gelatin-coated tissue culture flasks. 176 Cells were left to proliferate for 14 days at $37^{\circ} \mathrm{C}-5 \% \mathrm{CO}_{2}$ in DMEM-glutaMAX medium 177 supplemented with $10 \% \mathrm{FBS}, 50 \mathrm{mg} / \mathrm{ml}$ penicillin-streptomycin and $50 \mathrm{mg} / \mathrm{ml}$ fungizone. Medium 178 was renewed after 7 days, cells were passaged after 14 days and further cultured in DMEM179 glutaMAX with 10\% FBS. Two days later, FBS was reduced to 3\% and medium was supplemented 180 with the growth factor cocktail G5. All experiments were conducted 7 days later (DIV7).

\section{$181 \quad$ Npas4 induction analysis}

182 For Npas4 induction analysis, neurons and astrocytes at DIV7 were depolarized with 50mM 183 potassium chloride. Cell lysates were analyzed by Western blotting with the anti-Npas 4 antibody. 184 as described below.

\section{RNA extraction, transcriptome analysis and $q R T-P C R$}

186 Total RNA was extracted by TriPure Isolation Reagent according to the manufacturer's protocol. 187 RNA samples were suspended in DEPC-treated water and RNA concentration was measured (OD $188260 \mathrm{~nm}$ ) on BioSpec-nano spectrophotometer (Shimadzu Biotech). For microarray analysis, RNA 189 quality was evaluated by capillary electrophoresis using the Agilent 2100 Bioanalyzer instrument 190 with the Agilent RNA 6000 Nano Kit according to the manufacturer's instructions (Agilent, Santa 191 Clara, CA). $250 \mathrm{ng}$ of total RNA for each sample was amplified and labeled using GeneChip®WT 192 PLUS Reagent kit (Affymetrix) before being hybridized on GeneChip ${ }^{\circledR} M o u s e$ Transcriptome 1.0 193 Array, overnight at $45^{\circ} \mathrm{C}$. The chip was washed using an automated protocol on the GeneChip® 194 Fluidics Station 450 followed by scanning on a GeneChip® Scanner on Affymetrix microarray 195 platform (de Duve Institute, UCL, Brussels). 
196 For quantitative PCR, RNA samples were reversed transcribed using iScript cDNA Synthesis Kit and real time PCR was performed in an iCycler MyIQ2 multicolor-Real-Time PCR detection system using iQ SYBR Green supermix kit (Biorad). A standard curve was established for relative quantification with a fourfold dilution series (from 100 to 0,0097 ng) of a cDNA template mix. Relative quantification was calculated by the $2^{\Delta \Delta \mathrm{CT}}$ method, and results were normalized first to Gapdh expression and then normalized (percentage or fold) to the control condition. Primers used are depicted in Table 1 in Supplementary material.

\section{Western blotting}

204 Cells were solubilized and sonicated in lysis buffer (20\% Glycerol, 4\% SDS, $125 \mathrm{mM}$ Tris-HCl $205 \mathrm{pH}$ 6.8) containing a cocktail of proteases and phosphatases inhibitors. Mice were euthanized 206 (Ketamine/Xylazine injection) and brains were dissected after perfusion with ice cold sterile PBS. Cortices and hippocampi were isolated and quickly frozen in liquid nitrogen until use. Tissues were crushed using mortar pestle method. For brain protein extraction, samples were homogenized in RIPA buffer (1\% (w/v) NP40, 0.5\% (w/v) deoxycholic acid, 0.1\% (w/v) SDS, $150 \mathrm{mM} \mathrm{NaCl,} 1$ mM EDTA, $50 \mathrm{mM}$ Tris, $\mathrm{pH}$ 7.4) containing proteases and phosphatases inhibitors cocktail (Roche, Basel, Switzerland). The samples were clarified by centrifugation at 20,000 x g. Protein concentrations were determined with a BCA kit. Samples were prepared with NuPAGE LDS sample buffer (4x) and $50 \mathrm{mM}$ DTT and then heated for $10 \mathrm{~min}$ at $70^{\circ} \mathrm{C} .10$ to $40 \mu \mathrm{g}$ of proteins or $22 \mu \mathrm{l}$ of culture medium were loaded per well for migration followed by transfer onto PVDF or nitrocellulose membranes. For APP C-terminal fragments blotting, proteins were transferred on nitrocellulose $(0.1 \mu \mathrm{m})$. Membranes were blocked in nonfat dry milk (5\% in PBS, 0,1\% Tween$20)$ and immunoblotted with anti-APP NT (22C11, 1/500), anti-APP CT (Y188, 1/500), antiAPLP1 (1/1000), anti-APLP2 (1/1000) and anti-GAPDH (1/25000). Blots were revealed using

ECL and signal quantification was performed using GelQuant.NET software (BiochemLabSolutions.com).

\section{ImmunoCytoFluorescence (ICF)}

222 Neurons were grown at 100.000 cells/ $\mathrm{cm}^{2}$ per well on poly-L-lysine coated coverslips. Neurons 223 were rinsed with PBS and fixed for $15 \mathrm{~min}$ in PBS/4\% paraformaldehyde. Neurons were washed 224 again twice in PBS for $5 \mathrm{~min}$ and processed as described previously (Decock et al., 2016). Permeabilization and blocking steps were done in PBS/5\% skimmed milk/0.3\% Triton-X100 (M3TPBS); antibodies were incubated in PBS/5\% skimmed milk/0.1\% Triton-X100 (M1TPBS). Primary antibodies dilutions used: mouse anti-MAP2 (1/1000), rabbit anti-APP (Y188, 1/100) and rabbit anti-GAD65 (D5G2, 1/100). Secondary antibodies dilutions used: goat anti-mouse Alexa 230 Fluor®-647 (1/500). Images were acquired on Evos FL Auto microscope (Invitrogen) with GFP 231 (Alexa Fluor®-488 or native GFP), TxRed (Alexa Fluor®-568) and CY5 (Alexa Fluor®-647) 232 EVOS LED light cubes and analyzed with ImageJ software. For the quantification of signal area, $23310 \mathrm{X}$ or $20 \mathrm{X}$ magnification images were identically thresholded for APP+/+ and APP-/- or Ct and 
234 CRISPR-Npas4. Area of thresholded images was measured and normalized to the number of cells 235 counted by DAPI staining. For the quantification of the APP expression intensity, image 236 acquisition was performed using 40x objective coverslip-corrected (ThermoFischer Scientific, 237 AMEP4699) in GFP, CY5 (APP) and DAPI channels. A total of 12, 19 and 19 images were acquired to obtained 33, 46 and 51 neurons in the analysis (Figure 3B) for CRISPR control (Ct), Oligo2 and Oligo17 respectively. GFP channel images were first 8-bit transformed and thresholded to highlight only GFP staining. A region of interest (ROI) was delimited around GFP+ neurons in the GFP channel (green using "wand tool" in imageJ software and transposed to CY5 (APP) channel (blue). ROI mean intensity is measured using "Analyze" tool of ImageJ software.

\section{AICD and CRISPR/Cas9 lentiviral constructions and production}

244 We used a lentiviral vector-based approach to express AICD in neurons. AICD50 tagged at the cterminal part with hemagglutinin (HA) was cloned into pLenti CMV/TO Puro lentiviral vector (Addgene \#17482). pLenti CMV/TO Puro empty is used as control (Ct). We used a lentiviral vector-based approach to deliver the CRISPR-Cas9 system. We designed sgRNAs "Oligo2" and "Oligo17" to target App mouse gene (Gene ID: 11820), and sgRNA "CRISPR-Npas4" to target Npas4 mouse gene (Gene ID: 225872). sgRNAs were cloned in a lentiviral vector delivering sgRNA, SpCas9 and coexpressing eGFP (Addgene \#57818) according to author instructions (Heckl et al., 2014). The negative control (Ct) used was the lentiviral construct without sgRNA but expressing SpCas9 and eGFP. sgRNA sequences, scores and PAMs are provided in Table 2 in Supplementary material. Briefly, sgRNAs purchased at Sigma-Aldrich (St Louis, MO, USA) were designed using on/off-target score algorithm and cloned into the pL.CRISPR.EFS.GFP plasmid. Vectors were validated by sequencing (Beckman Coulter Genomics, UK), produced and purified HEK293-T cells in $10 \mathrm{~cm}$ dishes $\left(2 \times 10^{6}\right.$ cells/dish) with lentiviral CRISPR-Cas9 vectors, pCMVdR8.2 (Addgene\#12263) and pMD2.G (Addgene\#12259). After $48 \mathrm{~h}$, the supernatant was filtered and incubated with $1 / 3(\mathrm{v} / \mathrm{v})$ of LentiX ${ }^{\mathrm{TM}}$ Concentrator for $90 \mathrm{~min}$ on ice. The collected supernatant was centrifuged at $1500 \mathrm{xg}$ for $45 \mathrm{~min}$ at $4^{\circ} \mathrm{C}$, the pellet was resuspended in $20 \mu \mathrm{l}$ per dish of

261 Neurobasal ${ }^{\circledR}$ Medium and stored at $-80^{\circ} \mathrm{C}$ until use. Empty backbone of pL-CRISPR.EFS.GFP 262 was used as negative control $(\mathrm{Ct})$ in our studies.

263 Neurons were infected with lentiviruses CRISPR-Cas9 1 day after plating (DIV1). Typically, 20

$264 \mu \mathrm{l}$ of concentrated virus were used to infect 800.000 cells per well of 12 well plate dish. The 265 medium was completely changed after 24 hours and a half media change was carried out every 2-

2663 days thereafter. The neurons were harvested at 7 days in vitro (DIV7) or as indicated.

\section{Lentiviral toxicity assay}

268 Cell viability was measured by LDH release in the culture medium at DIV7 after lentiviral infection 269 using Cytotoxicity Detection kit (Sigma-Aldrich, St-Louis, MO, USA) according to the 270 manufacturer's instructions. Relative absorbance was measured at $490 \mathrm{~nm}$ using a VICTOR 
271 Multilabel Plate Reader (PerkinElmer, Richmond, VA, USA). Background LDH release was 272 determined in non-infected control cultures.

\section{$273 \quad$ Flow cytometry and cell sorting}

274 After DIV7, infected neurons were briefly rinsed with PBS and trypsinized for 2 min. Neurons 275 were mechanically dissociated and filtered through $70 \mu \mathrm{m}$ Falcon ${ }^{\mathrm{TM}}$ Cell Strainers in $50 \mathrm{ml}$ tube

276 containing FBS. Cells were pelleted by centrifugation at 1000xg for $5 \mathrm{~min}$ and resuspended in 277 PBS/1\% FBS/1mM EDTA. TO-PRO ${ }^{\text {TM}}$-3 Iodide (Thermo Fisher Scientific) was used to stain dead 278 cell and exclude them for the sorting. Cells were sorted using a BD FACSAria ${ }^{\mathrm{TM} I I I}$ cell sorter (BD 279 Biosciences, San Jose, CA) on the "Flow cytometry and cell sorting - CYTF" UCL platform. The 280 sort parameters used were the following: nozzle $100 \mu \mathrm{m}$, sheath pressure 20 psi, drop frequency $28130 \mathrm{kHz}$ and sort precision 16-32-0. Sample and collection tubes were maintained at $4^{\circ} \mathrm{C}$ throughout 282 the sort. GFP-negative and positive cells were harvested in PBS/1\% FBS/1mM EDTA and 283 centrifuged at 12000xg for $2 \mathrm{~min}$ and homogenized in TriPure Isolation Reagent for RNA 284 extraction.

\section{Glutamate and GABA measurements}

286 Glutamate and $\gamma$-aminobutyric acid (GABA) were measured in medium and in cells at DIV7. 287 Briefly, neurons were grown at 200.000 cells $/ \mathrm{cm}^{2}$ in 12 well plate culture dish. Media were 288 harvested, centrifuged to pellet cell's debris and supplemented with cocktail of proteases inhibitors and frozen at $-20^{\circ} \mathrm{C}$ until use. Cells were scratched in ice cold PBS and pelleted by centrifugation $290\left(12.000 \mathrm{xg}\right.$ for $3 \mathrm{~min}$ at $\left.4^{\circ} \mathrm{C}\right)$ then quickly frozen in liquid nitrogen and kept at $-80^{\circ} \mathrm{C}$ until use. For glutamate assay: Media were directly used as are. Cells were prepared according to the manufacturer protocol and measurement was normalized on protein content. For GABA ELISA assay: Media were directly used as are. Cells were lysed by 5 cycles of thawing and freezing in PBS and centrifuged at $12.000 \mathrm{xg}$ for $10 \mathrm{~min}$ at $4^{\circ} \mathrm{C}$. Supernatant was used for the quantification and normalized on protein content.

\section{Statistical analysis}

297 Microarray analysis: Raw data were analyzed using Bioconductor ( $\mathrm{R}$ environment). Robust 298 Multiarray Average (RMA) was used for background correction, normalization, probe level intensity calculation and probe set summarization. Gene expression values were compared between $300 \mathrm{APP}+/+$ and APP-/- neurons at different stage of development DIV3, DIV7 and E18 using the R301 Limma (Linear Models for MicroArray Data) package. Benjamini-Hochberg procedure was used 302 for multiple testing corrections. From raw data, only transcripts with an Entrez ID were kept in 303 order to facilitate the analysis. Gene set enrichment analysis was performed on differentially 304 expressed genes sets after the ROAST (Rotation gene set tests for complex microarray 305 experiments) (Wu et al., 2010) procedure to identify KEGG pathways modified in absence of APP 306 for all conditions (E18, DIV3 and DIV7). The data obtained have been deposited in NCBI's Gene 307 Expression Omnibus (Edgar et al., 2002) and are accessible through GEO Series accession number 308 GSE112847 (https://www.ncbi.nlm.nih.gov/geo/query/acc.cgi?acc=GSE112847). 
309 Otherwise, statistical analyses were performed using GraphPad Prism (GraphPad Software, San 310 Diego, CA). Gaussian distribution was assessed by Kolmogorov-Smirnov test (GraphPad Prism). 311 If the data follow normal distribution parametric test was applied. Otherwise non parametric test 312 was used. If two groups were compared, parametric Student's t-test or non-parametric Mann313 Whitney test were used. If more than two groups were compared, parametric ANOVA with post 314 hoc tests as indicated or non-parametric Kruskall-Wallis were used. $*^{*}, \mathrm{p}<0,05 ; * *, \mathrm{p}<0,01$; ***, $315 \mathrm{p}<0,001)$. The number of biological replicate $(\mathrm{n})$ analyzed is indicated in figure legends in the 316 number of independent experiment $(\mathrm{N})$. 


\section{Results}

\section{APP-dependent expression of Npas4 in differentiated primary neuron cultures}

320 Experiments were performed on primary neuron cultures according to the workflow described in the expression of APP family proteins and observed an increase in APP, APLP1 and APLP2 upon differentiation with a peak of expression at DIV 7-8 (Figure S1B-C), supporting an important role of APP protein family in neuronal maturation. No modifications of APLP1 or APLP2 levels and maturation were observed in APP-/- neurons when compared to $\mathrm{APP}+/+$ at any time point of differentiation studied (Figure S1D). Thus, results obtained here in APP-/- neurons can be related to the loss of APP and not to indirect effects resulting from up- or down-regulation of APLP1 or APLP2. Previous studies indicated that AICD is detectable inside the nucleus specifically at DIV6restricted. We checked for AICD production at DIV7 in total lysates of APP+/+, APP+/- and APP/- cultures (Figure S1E). AICD was readily detectable in APP $+/+$ neurons at but only at high exposure time, confirming that it is a transient peptide (Huysseune et al., 2007) with a restricted temporally expression pattern in primary neurons. AICD-dependent transcriptional regulation may therefore only occur within a defined time-period, around DIV7 (and not at DIV3).

To investigate this, we performed microarray experiments, which allow description of genomewide expression changes in APP +/+ and APP-/- primary cortical neurons at DIV3 (immature neuronal network), DIV7 (neuronal network with detectable AICD) and in E18 cortical tissue (summarized in Figure S1A). We used Affymetrix GeneChip ${ }^{\circledR M o u s e ~ T r a n s c r i p t o m e ~ 1.0 ~ A r r a y ~ a n d ~}$ carried out data analysis with the R-Limma (Linear Models for MicroArray Data) package (Ritchie et al., 2015). The chips used allow the profiling of coding and non-coding (lncRNA, miRNA, pseudogene...) gene expression as well as alternative splicing events. We ran each condition (E18, DIV3, and DIV7) in triplicate (3 chips used for each condition, from independent cultures). We focused here on differentially expressed coding genes, although data were collected for non-coding RNAs (not shown). Strikingly, the overall changes observed (fold changes) were moderate in all conditions (E18, DIV3 and DIV7). Few coding transcripts appear to be differentially expressed when the specific fold change (linear) is set at 1.25, 1.5 or 2 (Figure 1A). The Benjamini-Hochberg multiple correction test did not reveal any robust differential gene expression (adjusted p-value $<0,05$ ) except as expected for APP (positive control). Gene enrichment analysis was performed using ROAST (Rotation gene set test for complex microarray experiment) procedure to finally identify molecular interaction/reaction networks diagram (Kanehisa and Goto, 2000) also known as KEGG pathway altered in the absence of APP. The first five pathways (in terms of significance), the number of genes modified as well as their direction are shown in Figure 1B. Interestingly, ECM (extracellular matrix)-receptor interaction and Long-term potentiation pathways are modulated in absence of APP at DIV7. Cell-ECM interactions are mediated by transmembrane receptors and cell adhesion proteins, involved in adhesion, differentiation and maturation. Long term potentiation 
357 (LTP) is a major mechanism in memory formation and learning. Both of these pathways have been 358 associated to APP function (Caceres and Brandan, 1997; Seabrook et al., 1999; Puzzo et al., 2011).

359 To note, we did not measure any expression change (Supplementary Table 3) of genes identified 360 as AICD target genes (Pardossi-Piquard and Checler, 2012).

361 We decided to further select genes relevant to described APP cellular function in order to further investigate their regulation by APP. In a set of array (APP+/+, accession number GSM3089741 vs APP-/- accession number GSM3089744) from a primary neurons at DIV7, we noticed a downregulation of (IEGs) in APP-/- neurons (Figure 1C). Among them, the activity-dependent transcription factor, Npas4 (Neuronal PAS domain protein 4) was of particular interest. NPAS4 is a neuron-specific IEG, known to be regulated by neuronal activity and involved in synaptic plasticity and synaptic homeostasis. We confirmed by qPCR that the Npas 4 mRNA level was decreased at DIV7 in APP-/- neurons compare to APP+/+, but not at DIV3 nor in the cortex at E18 (Figure 1D). To note, the expression of other early genes (Egr1 and Egr3) previously reported to be involved in APP-dependent gene expression (Hendrickx et al., 2013) were not altered in our conditions (Figure S2A, S2B).

\section{$372 \quad$ Npas4 expression is AICD-independent}

373 Since transcription of APP target genes could involve AICD (Belyaev et al., 2010), which is

374 produced particularly at DIV7, we inhibited its release by treating neurons with $1 \mu$ M DAPT for 16

$375 \mathrm{~h}$, a well-described non-competitive $\gamma$-secretase inhibitor (Dovey et al., 2001). DAPT treatment

376 induced APP CTFs accumulation (Figure 2A), indicating that $\gamma$-processing thus AICD release were 377 inhibited under these conditions (Hage et al., 2014). DAPT treatment didn't decrease Npas4 378 expression in APP-/- primary neurons, but indeed increased it in APP+/+. To further address the 379 role of AICD in Npas4 regulation, we transduced primary neurons with a lentiviral vector 380 expressing the $50 \mathrm{C}$-terminal amino acids of APP (AICD) fused (C-terminus) to the hemagglutinin 381 tag (HA). AICD-HA is detectable in infected cells (Figure 2C), and AICD expression in APP-/382 neurons did not modify Npas4 mRNA levels (Figure 2D), confirming that AICD is not involved in 383 APP-dependent Npas4 transcriptional regulation. As some of the APP functions were found to rely 384 on its extracellular soluble fragment $(\operatorname{sAPP} \alpha)$, we tested whether the sAPP $\alpha$ can regulate Npas 4 385 expression per se. Neuronal cultures were treated with $20 \mathrm{nM}$ of human sAPP $\alpha$ for $16 \mathrm{~h}$ (Figure 386 2E) and Npas4 expression was measured by qPCR. Npas4 mRNA levels increased significantly 387 upon sAPP $\alpha$ addition in APP+/+ neurons, but not in APP-/- (Figure 2F). These data provide a 388 general insight into APP-dependent Npas4 transcription in neurons. (i) AICD release is not 389 involved in this process; (ii) APP soluble ectodomain ( $\mathrm{APP} \alpha$ ) regulates Npas4 expression, only in 390 a context where endogenous APP is expressed. Important to note, glial cells represent about $\sim 16 \%$ 391 of total cells in primary cultures, and can indirectly contribute to the mechanisms we observed. 392 However, absence of APP did not change the astrocytic pattern of primary cultures, and astrocytes 393 do note express readily detectable Npas4 levels (Figure S3A-B). Hence, our observations reflect 394 APP-dependent Npas4 regulation truly acting in neurons 
The APP-dependent transcriptional regulations we observed were subtle when compared to those reported in the literature, in line with the mild phenotype of APP knockout mice (Muller et al., 1994; Zheng et al., 1995). Although we did not observe compensation of APP loss by APLP overexpression in our model, APP-dependent gene regulations that appear in the close-up could be hidden in the long term or related to functional redundancies with other members of the APP family (Shariati and De Strooper, 2013). In this line, APP-/- mice brain phenotype is better unraveled by acute down-regulation of APP (Senechal et al., 2007). We decided therefore to knock-down the APP expression in APP+/+ neurons with a lentiviral-based CRISPR-Cas9 genome editing approach (Jinek et al., 2012), to test the consequence of acute APP knock-down on Npas4 expression. Nearly $\sim 50 \%$ of the cells in culture were infected under our conditions (Figure S4A-B) and no lentiviral toxicity was measured (Figure S4C). Only neuronal cells were infected, reflecting the tropism of the viral particles for neurons, and not for glial cells. APP expression was monitored by ICF (Figure 3A) and by measuring the intensity of APP signal in GFP-positive (infected) neurons (Figure 3B). APP was strongly decreased in neurons infected with viruses expressing the Oligo 2 and 17 sgRNA sequences targeting APP exon1 and exon 2, respectively, when compared to Ct. This was confirmed by Western blotting showing the APP expression specifically decreased by about $50 \%$. Importantly, APLP1 and 2 expressions were not altered in cultures infected with Oligo2 and Oligo17 lentiviruses (Figure $3 \mathrm{C}$ ), clearly indicating that off targets mechanisms - a major risk with CRISPR-Cas9 approaches, especially with homologous genes - are not observed in our experimental setup.

415 We decided to measure the expression of Npas4 after APP knock-down selectively in GFP-positive (knock-down) neurons. This was achieved by sorting GFP positive cells by flow cytometry. We used TO-PRO ${ }^{\text {TM}}-3$ staining as a viability marker to exclude dead cells from the analysis and set sorting parameters by using non-infected condition (no GFP) and neurons expressing GFP (GFP infected) as standards. Npas 4 mRNA levels were measured in these cells by qPCR (Figure 3D). Npas4 mRNA was readily decreased in neurons infected with Oligo2- and 17-expressing lentiviruses. Acute APP knock-down achieved with the CRISPR-Cas9 system in primary neurons resulted in the decrease in Npas 4 expression, confirming the APP-dependent Npas 4 transcriptional expression observed in APP deficient neurons.

\section{APP deficiency increases the markers of GABAergic transmission}

425 Down-regulation of Npas 4 expression in the absence of APP could reflect an impairment in neurite formation and/or synaptogenesis which may lead to deficient in basal neuronal activity. APP was reported to modulate neurite outgrowth and synapse formation (Priller et al., 2006; Young-Pearse et al., 2007; Tyan et al., 2012; Billnitzer et al., 2013) but the mechanisms by which APP modulates synapse formation and plasticity is poorly understood. We first analyzed neuronal arborization at 430 DIV7, when Npas4 expression is decreased. We monitored arborization by measuring the area of the neuron-specific microtubule associated protein2 (MAP2) signal per cell from DIV1 to DIV7 432 (Figure 4A). APP-/- neurons extend neurites and no difference was observed at DIV1 to DIV3. The 
433 absence of APP subtly (but significantly) increased MAP2 signal at DIV7 (Figure 4B), indicating 434 the importance of APP for proper neurite arborization.

435 Npas4 is involved in the fine tuning of excitatory/inhibitory homeostasis, by controlling the balance 436 of excitatory and inhibitory inputs on post-synaptic neurons (Lin et al., 2008; Bloodgood et al., 437 2013; Spiegel et al., 2014). This characterized by the type of neurotransmitter released: typically 438 glutamate for excitatory synapses and GABA for inhibitory synapses. We measured the amount of 439 GABA and glutamate released in medium and present in the cells at DIV7 (Figure 5A-B). The 440 concentration of GABA is increased by $83 \%$ in the medium of APP-/- neurons (Figure 5A), and no difference was observed for GABA measured in cells. Strikingly, we observed no significant change in glutamate concentration (cell or medium) in APP-/- neuronal cultures compare to $\mathrm{APP}+/+$ (Figure 5B). This is supported by the only slight qualitative modifications in glutamate responses measured by intracellular calcium imaging in APP-/- neurons (Figure S5). GABA is synthetized by the glutamate decarboxylase enzymes $\left(\mathrm{GAD}_{65}\right.$ and $\left.\mathrm{GAD}_{67}\right)$ that catalyze the decarboxylation of glutamate to GABA. GAD 65 synthesizes GABA for neurotransmission, and is therefore active at nerve terminals and synapses. By immunostaining, we observed that GAD $_{65}$ signal is increased in APP-/- neurons compared to APP+/+ neurons (Figure 5C). This is not caused by an increase in the relative number of GABAergic neurons in APP-/- cultures compare to APP $+/+$ (Figure S6), pointing to an increase in GAD65 cellular expression. To further address the effect of APP deficiency on GABAergic neurotransmission, we quantified the expression of the most prevalent GABA receptor subunit, GABARa1 expressed during neuronal development. We found GABARa1 to be slightly but significantly decreased in APP-/- neurons (Figure 5D), suggesting complex modifications of GABAergic neurotransmission. In summary, our results indicate that APP deficiency disturbs mainly GABAergic neurotransmission components with a little effect on the excitatory counterpart.

457 Finally, we evaluated whether impairments affecting GABAergic neurotransmission components 458 we observed in vitro in vitro could are relevant in the brain. We quantified the expression of GAD65 in cortices and hippocampi of 3 month old mice deficient for APP (APP-/-) compared to their wild-type counterpart (APP+/+). Consistent increase in GAD65 expression is observed both in cortex (Figure 6A) and in hippocampus (Figure 6B) of APP-/- mice; indicating that GABAergic neurotransmission component GAD65 is also affected in adult mouse brain and supporting our in vitro findings.

\section{Phenotype of NPAS4-deficient neurons mimics APP deficiency}

As for APP, we used the CRISPR-Cas9 approach in order to silence Npas4 and analyze whether NPAS4 deficiency could recapitulate a major trait observed in APP-/- neurons, i.e. imbalance of inhibitory transmission by the upregulation of GABA release. Given that (i) CRISPR-Cas9 editing is hard to evaluate by quantifying mRNAs expression, (ii) that available antibodies poorly detect 
471 silencing resulted in a decrease in NPAS4 by approximately 50\% (Figure 7A), a similar extent to 472 that observed of mRNAs in APP-/- neurons at DIV7 (Figure 1D). This downregulation of NPAS4 473 is not due to a lentiviral toxic effect (Figure S4C). Strikingly, like for APP-deficient neurons 474 (Figure 5C), NPAS4-deficient neurons showed an increase in GAD65 staining (Figure 7B-C), 475 GAD65 protein expression (Figure 7D), and GABA release in the medium (Figure 67C) when 476 compared to control neurons. We measured the expression of GABA receptor subunit alpha 1 477 (GABARa1) at DIV7 and observed, like in APP-/- primary neurons, a decrease in protein 478 expression after Npas4 knockdown (Figure 7D). 


\section{Discussion}

481 One major APP function is to control synaptic formation, transmission and plasticity (Muller et al., 482 2017). We showed here that APP deficiency in cortical neurons impairs the balance between 483 excitatory and inhibitory synaptic markers, and that this process relies on the activity-dependent 484 transcription factor NPAS4. We initially identified the Npas4 IEG as a potential APP target gene 485 by a non-biased transcriptome profiling approach. The APP-dependent regulation of Npas 4 486 expression involves its extracellular domain (sAPP $\alpha$ ) but not AICD. APP appears to exert a fine 487 tuning of excitatory/inhibitory synaptic inputs in neurons and its absence enhances, through the 488 downregulation of Npas4, inhibitory GABAergic transmission.

\section{APP-dependent expression of Npas4 in differentiated neuronal culture}

490 The transcriptome analysis of APP+/+ vs. APP-/- neurons at embryonic day 17 (E18-DIV0) and at 491 different stages of primary cortical neuron differentiation (DIV3-DIV7) indicated that the 492 transcriptional changes in the absence of APP were moderate. This unexpected result is however 493 in line with a comparative transcriptome study of APP family members in the adult mouse cortex 494 (Aydin et al., 2011). One possible explanation to the subtle effects of APP deficiency on the 495 transcriptome can be functional compensation by APLPs (Shariati and De Strooper, 2013). 496 However, we did not measure any changes in APLP1 and APLP2 expression in our APP-/- models in agreement with previous observations total brain extracts (Zheng et al., 1995) or in primary 498 cortical neurons (White et al., 1998). Transcriptional modifications we measured are thus related to APP per se. APP-dependent transcriptional regulations are subtle, and likely to act by fine-tuning

500 classes of gene involved in neuronal pathway rather than single target genes. In addition, we found 501 that none of the APP/AICD target genes were differentially expressed in APP-/- neurons at DIV3502 DIV7 or at E18 (See Supplementary Table 3). The identification of AICD-dependent gene 503 expression stemmed from studies carried out in an array of in vitro- and -to a lesser extent- in vivo 504 models (for review see Pardossi-Piquard and Checler, 2012; Grimm et al., 2013). Some of these 505 findings were confirmed or debated in subsequent investigations (Hebert et al., 2006; Chen and 506 Selkoe, 2007; Waldron et al., 2008; Aydin et al., 2011). AICD-dependent gene transcription and 507 how it relates to APP function appears thus, if not controversial, scarcely understood.

508 We found that the expression of Npas4, an activity-dependent IEG, is downregulated in the absence 509 of APP and particularly at DIV7. Npas4 downregulation was observed it in APP-/- primary neurons 510 and upon acute APP knock-down by a CRISPR-Cas9 approach (Figure 3D), establishing a causal 511 relation between APP and Npas4 transcription. Furthermore, APP-dependent Npas4 expression at 512 DIV7 does not rely on AICD release, although DIV7 corresponds to the differentiation stage where 513 AICD is readily produced by neurons (Kimberly et al., 2005). Previous studies indicated that 514 regulation of some APP target genes does not require the generation of AICD (Hicks et al., 2013). 515 Quite strikingly, DAPT treatments, used to block AICD production in our setup, increased Npas4 516 expression in APP+/+ neurons. This effect might imply that inhibition of $\gamma$-secretase increases the 517 neuronal activity in an APP-dependent manner (APP-/- neurons showed no modification of Npas4 
518 expression after treatment). In line with this, $\gamma$-secretase inhibition was shown to increase 519 excitatory postsynaptic currents (EPSCs) (Priller et al., 2006; Restituito et al., 2011). Further 520 investigations are required to understand this observation. However, one hypothesis could be that 521 the loss of A $\beta$ underlies the DAPT effects we observed. Several studies reported that A $\beta$ depresses 522 AMPA- and NMDA-receptor mediated currents and EPSCs in neurons arguing toward a negative 523 feedback of A $\beta$ on synaptic transmission (Kamenetz et al., 2003; Snyder et al., 2005; Hsieh et al., 524 2006). This feedback is not possible in a APP-/- background. Alternatively, studies indicated that 525 inhibition of $\gamma$-secretase induces an increase of production of sAPP $\alpha$ (Chen et al., 2015). In that 526 case, increased activity related to increased sAPP $\alpha$ production would corroborate the results we 527 obtained on Npas 4 expression by treating neurons with sAPP $\alpha$. We also found that sAPP $\alpha$ effects 528 on Npas4 expression are observed only in APP+/+ and not in APP-/- background. This observation 529 indicates that (i) the transcriptional effects of $\operatorname{SAPP} \alpha$ require the presence of endogenous APP 530 holoproteins (ii) homophilic ectodomain interactions are likely to be involved. Soluble APP has 531 been shown to rescue many traits of APP-deficient mice (Ring et al., 2007; Weyer et al., 2014) and 532 was suggested to promote its physiological effects by interaction with APP holoprotein (Milosch 533 et al., 2014; Deyts et al., 2016).

534 Alteration of GABAergic inputs in APP deficient neurons are related to Npas4 535 downregulation

536 In the absence of APP, we observed an increase in neuronal outgrowth and GAD65 signal, as well as increased GABA release in the medium. To note, Npas 4 knockdown mimics APP deficiency on GAD65 levels and GABA measurements (Figure 8). This supports the hypothesis APP regulates the fine-tuning inhibitory synaptic transmission in the neuronal network through NPAS4. First, this is in agreement with very recent work showing that APP regulates GABAergic neurotransmission during neuronal differentiation (Doshina et al., 2017). In vivo studies evidenced increased GABA levels in the brain of APP-/- mice (Lee et al., 2010). Secondly, the finding that APP-dependent neuronal processes are mediated by NPAS4 is relevant to experimental evidences reported in previous studies. NPAS4 possesses unique features among the IEGs (Sun and Lin, 2016): (i) it is only expressed in neurons; (ii) it is activated selectively by neuronal activity; (iii) it has been shown to be important to shape glutamatergic and GABAergic synaptic inputs. NPAS4 is implicated in a transcriptional program that regulates neuronal firing responses to excitatory transmission by enhancing inhibition (Lin et al., 2008), and is critical for the homeostatic mechanisms that keep neuronal firing in response to stimuli within normal levels (Spiegel et al., 2014). Increasing the excitability of a set of neurons leads to changes in both their input and axonal synapses. NPAS4 is necessary for modulating the inputs synapses but not the axonal synapses of these neurons (Sim et al., 2013). NPAS4 is induced in excitatory neurons, where it promotes increased numbers of inhibitory synaptic inputs. (Spiegel et al., 2014). Altogether, these specific functions of NPAS4 correlate well with our main observation that APP-dependent Npas4 expression is related to the upregulation of the GABAergic system in APP-deficient neurons. This is not restricted to primary 
The overall effect of APP deficiency of neuronal network activity and synaptic transmission needs further neurophysiological investigations that are beyond the scope of the present study. Important points must be kept in mind here. First, GABAergic transmission shifts from excitatory to inhibitory during development (Ben-Ari, 2002), and our findings should be evaluated by electrophysiological recordings in mature neurons. For instance, we found that the level of GABA receptor subunit alpha 1 (GABAR $\alpha 1$ ) was diminished in the absence of APP and in NPAS4deficient neurons. This is in agreement with recent study showing that GABAR $\alpha 1$ is particularly decrease in hippocampus of APP-/- mice (Chen et al. 2017) correlating with a decrease in IPSC amplitude. But on the other hand, it suggests that increases in GABA release in APP-deficient models may not result in a net increase of inhibitory transmission, or at least there is a complex modulation of neuronal response to GABA.

\section{Possible relevance to the AD pathophysiology}

569 APP plays a central role in the onset and progression of AD by releasing the A $\beta$ peptide, but, APP deficiency is more difficult to correlate to the pathology. Still, it is admitted that impairment of APP function per se, either caused by FAD mutations or upon ageing, may contribute to neuronal dysfunction occurring in the disease. For instance, the phenotype of APP deletion in the CNS is age-dependent (Priller et al., 2006). Upon aging, impairments in learning and memory associated with deficits in LTP are observed in APP-deficient mice (Ring et al., 2007). The role of APP in maintaining spine architecture is supported by the reduction in dendritic length and branching as well as in total spine density in old APP-deficient mice (Lee et al., 2010; Tyan et al., 2012). A severe decrease in metabolic activity was also observed in presynaptic densities of APP KO animals (Lassek et al., 2017). This is an important feature, because bioenergetics and metabolic activity are fueling the synthesis of neuromediators (glutamate and consequently GABA), and providing energy supply and calcium buffering essential for synaptic function and plasticity.

Significantly lower levels of GABA and glutamate were measured in the temporal cortex of AD patients, pointing to deficient synaptic function and an imbalance in neuronal excitatory/inhibitory transmission (Gueli and Taibi, 2013). These observations unambiguously support changes in neurotransmission in $\mathrm{AD}$ (and even in ageing brain), but the mechanisms underlying this process are hardly understood. Here we found that neuronal activity by itself, sensed by the NPAS4 IEG, reshapes synaptic GABAergic inputs on neurons, in line with recently reported modifications of GABA transmission in AD models (Doshina et al., 2017). Very interestingly, NPAS4 expression decreases along with AD progression, particularly at Braak NFT stages (I-II) corresponding to lesions developed in transentorhinal/entorhinal cortex (Miyashita et al., 2014). Downregulation of GABAergic transmission could also underlie the increased risk for unprovoked seizures observed in individuals with $\mathrm{AD}$ compared to non-demented individuals of the same age (Friedman et al., 2012).

593 In conclusion, our main observation that APP deficiency in neurons is integrated by the activity594 dependent NPAS4 IEG to further re-modulate inhibitory and excitatory neuronal inputs, provides 
bioRxiv preprint doi: https://doi.org/10.1101/504340; this version posted December 21, 2018. The copyright holder for this preprint (which was not certified by peer review) is the author/funder. All rights reserved. No reuse allowed without permission.

595 new insight to understand the role of APP in synaptic activity, but also a mechanistic frame to 596 further explore the impairments of network activity in AD. 


\section{Figure 1: APP-dependent expression of Npas4 in young differentiating neuronal culture}

600 Summary of transcriptome analysis performed with the GeneChip ${ }^{\circledR}$ Mouse Transcriptome Array 6011.0 (Affymetrix). Data were processed in triplicate (3 independent cultures) for each experimental 602 time point (E18; DIV3; DIV7). Non-coding transcripts and alternative splicing products are 603 detected by these arrays, but only transcripts of coding transcripts have been considered here. A) 604 Number of up-and down-regulated coding transcript in APP-/- vs. APP+/+ primary neurons at E18, 605 DIV3 and DIV7. Linear fold changes have been set at 1,25, 1,5 and 2. B) KEGG pathway analysis 606 (http://www.genome.jp/kegg/pathway.html) at E18, DIV3, DIV7 (APP-/- vs. APP+/+) to identify networks molecular pathways (or interaction networks) in which differentially expressed genes are clustered. The five most modified pathways are displayed for each time point, with the number of genes potentially up-or down-regulated. C) Immediate Early Genes (IEGs) expression in APP-/vs. APP+/+ primary neurons at DIV7 and their respective fold change (APP-/- vs APP+/+) in microarray analysis at DIV7. D) Neuronal PAS 4 domain (Npas4) mRNA level was measured by

612 qPCR at E18, DIV3 and DIV7 $(n=6, N=3)$. Results (mean \pm SEM) are expressed as percentage of controls $(\mathrm{APP}+/+)$. n.s. $=$ non-significant, $* \mathrm{p}=0.0242$, Student's t-test.

\section{Figure 2: APP metabolites but not AICD regulate Npas4 expression}

615 A) Western blotting analysis of APP and C-terminal fragments (CTFs and AICD) in cortical neurons after $1 \mu \mathrm{M}$ DAPT treatment for $16 \mathrm{~h}$ at DIV7. B) Quantification by qPCR of Npas $4 \mathrm{mRNA}$ in APP+/+ or in APP-/- neurons at DIV7 treated with $1 \mu \mathrm{M}$ DAPT for $16 \mathrm{~h}(\mathrm{n}=7, \mathrm{~N}=4)$. Results are expressed as percentage of control $(\mathrm{Ct})$ (mean \pm s.e.m.). ${ }^{*} \mathrm{p}=0.0216$, n.s. $=$ non-significant, infection in cells with control or AICD-HA expressing vectors. Total cell lysate was analyzed with anti-HA antibody. D) Quantification by qPCR of Npas4 mRNA in neurons at DIV7 infected with lentiviral vector expressing AICD-HA $(n=6, N=2)$. Results are expressed as percentage of control (Ct) (mean \pm s.e.m). n.s.= non-significant, Student-t test. E) Medium of sAPP $\alpha$ treated APP $+/+$ or APP-/- neurons was subjected to western blotting analysis using anti-human APP antibody (clone WO2) to detect the exogenous human sAPP $\alpha$ (h sAPP $\alpha$ ) and using anti-mouse APP antibody (clone $22 \mathrm{C} 11)$ to detect both endogenous and exogenous sAPP $\alpha(\mathrm{h}+\mathrm{m} \operatorname{sAPP} \alpha)$. Medium was collected after $16 \mathrm{~h}$ of treatment. F) Quantification by qPCR of Npas 4 mRNA level in APP+/+ $(\mathrm{n}=8, \mathrm{~N}=4)$ or in APP-/- neurons at DIV7 treated with $20 \mathrm{nM} \mathrm{sAPP} \alpha$ for $16 \mathrm{~h}(\mathrm{n}=6, \mathrm{~N}=3)$. Results are expressed as percentage of control $(\mathrm{Ct})\left(\right.$ mean \pm s.e.m.). ${ }^{*} \mathrm{*}=0.0055$, n.s. $=$ non-significant, Student-t test.

\section{$630 \quad$ Figure 3: Decreased Npas4 expression in APP-silenced primary neurons}

631 APP was knock-down by CRISPR-Cas9 approach in primary neurons cultures. A) Cortical neurons 632 were infected at DIV1 with lentiviruses expressing sgRNAs (Oligo2, Oligo17) or no sgRNA (Ct), 633 SpCas9 and GFP. Cultures were immunostained for MAP2 (red), APP (blue) and DAPI (light blue) 634 at DIV7. Arrowheads indicate the position of GFP-positive (infected) neurons in each condition. 
635 Scale bar: $100 \mu \mathrm{m}$. B) Quantification of APP signal in GFP-positive neurons. At least 33 neurons 636 were quantified in two independent experiments for each condition ( $n=33 \mathrm{~N}=2)$. Results (mean \pm 637 SEM) are given as percentage of control $(\mathrm{Ct})$. \#\#\#p<0,001 (Ct vs Oligo 2) ***p<0,001 (Ct vs 638 Oligo17); Kruskal-Wallis test and Dunn's multiple comparison test. C) Left panel. Representative 639 Western blots showing APP, APLP1, APLP2 and GAPDH protein level in cortical neurons at DIV7 640 infected in the same conditions. NI = non-infected. Right panel, quantification of APP expression 641 measured by Western blotting. Results (mean \pm SEM) are given as percentage of control $(\mathrm{Ct})$. $642 * * * \mathrm{p}<0,001$ (Ct vs Oligo17), \#\#\#p<0,001 (Ct vs Oligo 2), ANOVA and Bonferroni's multiple 643 comparison test ( $n=6, N=3$ ). D) Sorting of GFP-expressing neurons (FACS). Scatter plots (FSC vs. 644 SSC, left panels) of non-infected and GFP-expressing cells are shown. Dot plots (TOPRO-3, far 645 red vs. GFP, right panels) were used to gate (green rectangle) GFP-positive/TOPRO-3 negative 646 cells. RNA was extracted from these cells and Npas4 mRNA level was quantified by qPCR. Results 647 were obtained from pooled samples (4 wells of $4 \mathrm{~cm}^{2}$ each) for each condition (Ct, Oligo2 and 648 Oligo17). Quantification were carried out on 2 independent experiments $(\mathrm{N}=2)$. Results (mean \pm 649 SEM) are expressed as percentage of $\mathrm{Ct}$.

650 Figure 4: Altered neurites arborization of APP deficient neurons during in vitro maturation

651 A) Cortical APP+/+ or APP-/- were stained against the neuron-specific marker MAP2 and the 652 nuclear dye DAPI at different stages of maturation (DIV1-2-3 and DIV7). Scale bar: 400 $\mu \mathrm{m}$. B) 653 Quantification of MAP2 signal area normalized to the number of neurons at DIV1, 2, 3 and 7. 654 Quantifications were from 3 fields of at least 6 coverslips from APP $+/+$ and APP-/- neurons, in 655 three independent experiments $(\mathrm{N}=3)$. Results (mean $\pm \mathrm{SEM}$ ) are expressed as percentage of 656 control $(\mathrm{APP}+/+)$. ${ }^{*} \mathrm{p}=0,0293$, Mann-Whitney test.

A) Quantification of $\gamma$-amino butyric acid (GABA) in culture medium and cell extracts of APP $+/+$ and APP-/- primary neurons at DIV7. Results (mean \pm SEM) are expressed as percentage of APP $+/+(n=20, N=3) .{ }^{*} \mathrm{p}=0,0024$, n.s.= non-significant, Student-t test. B $)$ Quantification of glutamate in culture medium and cell extracts of APP $+/+$ and APP-/- neurons at DIV7. Results $($ mean \pm SEM) are expressed as percentage of APP $+/+(n=16, N=3)$. n.s.= non-significant, Studentt test. C) Cortical APP+/+ and APP-/- neurons at DIV7 were immunostained against the neuronspecific marker MAP2 and glutamate decarboxylase 65 (GAD65). Quantification of GAD65 signal area (5 fields per coverslip) was normalized to the number of cells. At least 2 coverslips were quantified for each group (APP $+/+$ and APP-/-) in two independent experiments $(\mathrm{N}=2)$. Results $($ mean \pm SEM) are given as percentage of control (APP $+/+)$. Scale bar: $200 \mu \mathrm{m} .{ }^{*} \mathrm{p}=0.0220$, Mann- 
671 expression. Results (mean $\pm \mathrm{SEM}$ ) are expressed as percentage of $\mathrm{Ct}(\mathrm{n}=5, \mathrm{~N}=2) .{ }^{*} \mathrm{p}=0.0197$, 672 Student's t-test.

\section{Figure 6: GAD65 expression in cortex and hippocampus of adult mice}

674 A) Left panel Western blot analysis of GAD65 and GAPDH expression in cortex of 3 month old $675 \mathrm{APP}+/+$ and APP-/- mice (N=5). Right panel Quantification of GAD65 was normalized to GAPDH 676 expression. Results (mean $\pm \mathrm{SEM}$ ) are expressed as percentage of $\mathrm{APP}+/+(\mathrm{N}=5)$. ${ }^{*} \mathrm{p}=0.0166$, 677 Student's t-test. B) Left panel Western blot analysis of GAD65 and GAPDH expression in 678 hippocampus of 3 month old $\mathrm{APP}+/+$ and APP-/- mice ( $\mathrm{N}=5)$. Right panel Quantification of 679 GAD65 was normalized to GAPDH expression. Results (mean \pm SEM) are expressed as percentage 680 of APP $+/+(\mathrm{N}=5) .{ }^{*} \mathrm{p}=0.0404$, Student's t-test.

682 Figure 7: Npas4 silencing by CRISPR-Cas9 mimicks cell phenotype observed in APP 683 deficient neurons.

684 Changes on inhibitory (GABA) synapses was analyzed after Npas4 silencing A) Left panel. 685 Cortical neurons infected with CRISPR-Cas9 lentivirus targeting Npas4 gene (CRISPR-Npas4) 686 show reduced NPAS4 levels as measured by Western blotting after membrane depolarization with $68750 \mathrm{mM}$ potassium chloride $(\mathrm{KCl})$. Control viruses without sgRNA were used as controls $(\mathrm{Ct})$. Note 688 that NPAS4 is barely detectable in non-depolarized neurons $(\mathrm{Ct})$. Right panel. Quantification of NPAS4 protein level after 2, 3 and $4 \mathrm{~h}$ of $\mathrm{KCl}$ depolarization. Results (mean $\pm \mathrm{SEM}$ ) are expressed 690 as percentage of non-treated controls $\mathrm{Ct}(\mathrm{N}=2)$. ${ }^{* * *} \mathrm{p}<0,0001$ Student's t-test. B) Cortical neurons 691 infected with CRISPR-Npas4 lentiviruses at DIV1 were immunostained against MAP2 and 692 glutamate decarboxylase 65 (GAD65) at DIV7. Quantification of GAD65 signal was normalized 693 to the number of cells (5 fields per coverslip, 2 coverslips for each genotype in two independent 694 experiments, $\mathrm{N}=2$ ). Results (mean $\pm \mathrm{SEM}$ ) are given as percentage of control $(\mathrm{Ct})$. Scale bar: $695200 \mu \mathrm{m}$. ** $\mathrm{p}=0.0024$. Mann-Whitney test. C) Quantification of $\gamma$-amino butyric acid (GABA) in 696 culture medium at DIV7 of infected control neurons (Ct) and CRISPR-Npas4 infected neurons. 697 Results (mean \pm SEM) are expressed as percentage of $\mathrm{Ct}(\mathrm{n}=5, \mathrm{~N}=2)$. ${ }^{*} \mathrm{p}=0,0146$, Student- $\mathrm{t}$ test. 698 D) Neurons harvested at DIV7 and cell extracts analyzed by Western blotting for GABAR $\alpha 1$, 699 GAD65 and GADPH expression. Quantification of GABAR $\alpha 1$ and GAD65 were normalized to 700 GAPDH expression. Results (mean \pm SEM) are expressed as percentage of $\mathrm{Ct}(\mathrm{n}=8, \mathrm{~N}=3)$. $701 * \mathrm{p}=0.049,{ }^{*} \mathrm{p}=0.0247$, Student's t-test. 


\section{Abbreviations}

705 A $\beta$, amyloid- $\beta$ peptide; AChE, acetylcholinesterase; AD, Alzheimer's disease; AICD, APP 706 intracellular domain; APP, Amyloid Precusor Protein; APLP1, APP-like Protein1; APLP2, APP707 like Protein 2; PPD, paired pulse depression; CRISPR, clustered regularly interspaced short 708 palindromic repeats; DAPT, N-[N-(3,5-Difluorophenacetyl)-L-alanyl]-S-phenylglycine t-butyl 709 ester; ECM, extracellular matrix; GABARa1, GABA(A) receptor subunit alpha-1; GAD65, 710 glutamate decarboxylase 65; GRIK1, glutamate ionotropic receptor kainate type subunit-1; IEG, 711 immediate early gene; KA, kainic acid; LTP, long term potentiation; NPAS4, neuronal PAS domain 712 protein 4; sAPP $\alpha$, soluble APP alpha.

\section{Acknowlegments}

715 pLenti CMV/TO Puro empty (w175-1) was a gift from Eric Campeau \& Paul Kaufman (Addgene 716 plasmid \#17482). pL-CRISPR.EFS.GFP (Addgene plasmid \# 57818) and pL-CRISPR.EFS.tRFP

717 (Addgene plasmid \# 57819) were a gift from Benjamin Ebert. pCMV delta R8.2 (Addgene plasmid 718 \# 12263) and pMD2.G (Addgene plasmid \# 12259) were a gift from Didier Trono. We thank 719 Jerome Ambroise for insight and technical support in the analysis of microarray data. We thank 720 Nicolas Dauguet for the cell cytometry sorting of the neurons. We thank Devkee Mahesh Vadukul 721 for her critical and linguistic revision of the manuscript. 


\section{Author contributions}

724 R.O. and P.K.C. designed the research study; R.O. conducted the experiments, with the help of

725 B.T., S.C., C.V., A.D., N.P. and F.P. Intracellular calcium measurement were designed and 726 performed with the help of P.D. and M.V. All the authors analyzed data. R.O. and P.K.C. wrote 727 the manuscript with the inputs of S.S., N.P. and J.N.O. All the authors have read and approved the 728 final manuscript.

\section{$729 \quad$ Funding}

730 This work was supported by the Belgian Fonds pour la formation à la recherche dans l'industrie et 731 1'agriculture (FRIA-FNRS), the Interuniversity Attraction Pole Programme-Belgian Sate-Belgian 732 Science Policy (IAP-P7/16 and IAP-P7/13), The Belgian Fonds de la Recherche Scientifique 733 Médicale (FRSM), the Queen Elisabeth Medical Foundation (FMRE), the Fondation pour la 734 Recherche sur la Maladie d'Alzheimer (SAO/FRA) and by the Action de Recherche Concertée 735 (ARC 14/19-059)

\section{Conflict of interest statement}

737 The authors confirm that there are no conflicts of interest. 


\section{References}

Alberini, C.M. (2009). Transcription factors in long-term memory and synaptic plasticity. Physiol Rev 89(1), 121-145. doi: 10.1152/physrev.00017.2008.

Aydin, D., Filippov, M.A., Tschape, J.A., Gretz, N., Prinz, M., Eils, R., et al. (2011). Comparative transcriptome profiling of amyloid precursor protein family members in the adult cortex. $B M C$ Genomics 12, 160. doi: 10.1186/1471-2164-12-160.

Belyaev, N.D., Kellett, K.A., Beckett, C., Makova, N.Z., Revett, T.J., Nalivaeva, N.N., et al. (2010). The transcriptionally active amyloid precursor protein (APP) intracellular domain is preferentially produced from the 695 isoform of APP in a \{beta\}-secretase-dependent pathway. $J$ Biol Chem 285(53), 41443-41454. doi: 10.1074/jbc.M110.141390.

Ben-Ari, Y. (2002). Excitatory actions of gaba during development: the nature of the nurture. Nat Rev Neurosci 3(9), 728-739. doi: 10.1038/nrn920.

Billnitzer, A.J., Barskaya, I., Yin, C., and Perez, R.G. (2013). APP independent and dependent effects on neurite outgrowth are modulated by the receptor associated protein (RAP). J Neurochem 124(1), 123-132. doi: 10.1111/jnc.12051.

Bloodgood, B.L., Sharma, N., Browne, H.A., Trepman, A.Z., and Greenberg, M.E. (2013). The activitydependent transcription factor NPAS4 regulates domain-specific inhibition. Nature 503(7474), 121 125. doi: 10.1038/nature12743.

Born, H.A., Kim, J.Y., Savjani, R.R., Das, P., Dabaghian, Y.A., Guo, Q., et al. (2014). Genetic suppression of transgenic APP rescues Hypersynchronous network activity in a mouse model of Alzeimer's disease. J Neurosci 34(11), 3826-3840. doi: 10.1523/JNEUROSCI.5171-13.2014.

Caceres, J., and Brandan, E. (1997). Interaction between Alzheimer's disease beta A4 precursor protein (APP) and the extracellular matrix: evidence for the participation of heparan sulfate proteoglycans. $J$ Cell Biochem 65(2), 145-158.

Chen, A.C., Kim, S., Shepardson, N., Patel, S., Hong, S., and Selkoe, D.J. (2015). Physical and functional interaction between the alpha- and gamma-secretases: A new model of regulated intramembrane proteolysis. J Cell Biol 211(6), 1157-1176. doi: 10.1083/jcb.201502001.

Chen, A.C., and Selkoe, D.J. (2007). Response to: Pardossi-Piquard et al., "Presenilin-Dependent Transcriptional Control of the Abeta-Degrading Enzyme Neprilysin by Intracellular Domains of betaAPP and APLP." Neuron 46, 541-554. Neuron 53(4), 479-483. doi: 10.1016/j.neuron.2007.01.023.

Chen, M., Wang, J., Jiang, J., Zheng, X., Justice, N.J., Wang, K., et al. (2017). APP modulates KCC2 expression and function in hippocampal GABAergic inhibition. Elife 6. doi: 10.7554/eLife.20142.

Cousins, S.L., Hoey, S.E., Anne Stephenson, F., and Perkinton, M.S. (2009). Amyloid precursor protein 695 associates with assembled NR2A- and NR2B-containing NMDA receptors to result in the enhancement of their cell surface delivery. J Neurochem 111(6), 1501-1513. doi: 10.1111/j.14714159.2009.06424.x.

Decock, M., Stanga, S., Octave, J.N., Dewachter, I., Smith, S.O., Constantinescu, S.N., et al. (2016). Glycines from the APP GXXXG/GXXXA Transmembrane Motifs Promote Formation of Pathogenic Abeta Oligomers in Cells. Front Aging Neurosci 8, 107. doi: 10.3389/fnagi.2016.00107.

Deyts, C., Thinakaran, G., and Parent, A.T. (2016). APP Receptor? To Be or Not To Be. Trends Pharmacol Sci 37(5), 390-411. doi: 10.1016/j.tips.2016.01.005.

Doshina, A., Gourgue, F., Onizuka, M., Opsomer, R., Wang, P., Ando, K., et al. (2017). Cortical cells reveal APP as a new player in the regulation of GABAergic neurotransmission. Sci Rep 7(1), 370. doi: 10.1038/s41598-017-00325-2.

Dovey, H.F., John, V., Anderson, J.P., Chen, L.Z., de Saint Andrieu, P., Fang, L.Y., et al. (2001). Functional gamma-secretase inhibitors reduce beta-amyloid peptide levels in brain. J Neurochem 76(1), 173 181. 
788

789

790

791

792

793

794

795

796

797

798

799

800

801

802

803

804

805

806

807

808

809

810

811

812

813

814

815

816

817

818

819

820

821

822

823

824

825

826

827

828

829

830

831

832

833

834

835

836

837

Fitzjohn, S.M., Morton, R.A., Kuenzi, F., Davies, C.H., Seabrook, G.R., and Collingridge, G.L. (2000). Similar levels of long-term potentiation in amyloid precursor protein -null and wild-type mice in the CA1 region of picrotoxin treated slices. Neurosci Lett 288(1), 9-12.

Freude, K.K., Penjwini, M., Davis, J.L., LaFerla, F.M., and Blurton-Jones, M. (2011). Soluble amyloid precursor protein induces rapid neural differentiation of human embryonic stem cells. J Biol Chem 286(27), 24264-24274. doi: 10.1074/jbc.M111.227421.

Friedman, D., Honig, L.S., and Scarmeas, N. (2012). Seizures and epilepsy in Alzheimer's disease. CNS Neurosci Ther 18(4), 285-294. doi: 10.1111/j.1755-5949.2011.00251.x.

Grimm, M.O., Mett, J., Stahlmann, C.P., Haupenthal, V.J., Zimmer, V.C., and Hartmann, T. (2013). Neprilysin and Abeta Clearance: Impact of the APP Intracellular Domain in NEP Regulation and Implications in Alzheimer's Disease. Front Aging Neurosci 5, 98. doi: 10.3389/fnagi.2013.00098.

Gueli, M.C., and Taibi, G. (2013). Alzheimer's disease: amino acid levels and brain metabolic status. Neurol Sci 34(9), 1575-1579. doi: 10.1007/s10072-013-1289-9.

Guo, Q., Wang, Z., Li, H., Wiese, M., and Zheng, H. (2012). APP physiological and pathophysiological functions: insights from animal models. Cell Res 22(1), 78-89. doi: 10.1038/cr.2011.116.

Hage, S., Marinangeli, C., Stanga, S., Octave, J.N., Quetin-Leclercq, J., and Kienlen-Campard, P. (2014). Gamma-secretase inhibitor activity of a Pterocarpus erinaceus extract. Neurodegener Dis 14(1), 3951. doi: 10.1159/000355557.

Hendrickx A, Pierrot N, Tasiaux B, Schakman O, Brion JP, Kienlen-Campard P, De SC, Octave JN (2013) Epigenetic Induction of EGR-1 Expression by the Amyloid Precursor Protein during Exposure to Novelty. PLoS One 8:e74305.

Hebert, S.S., Serneels, L., Tolia, A., Craessaerts, K., Derks, C., Filippov, M.A., et al. (2006). Regulated intramembrane proteolysis of amyloid precursor protein and regulation of expression of putative target genes. EMBO Rep 7(7), 739-745. doi: 10.1038/sj.embor.7400704.

Heckl, D., Kowalczyk, M.S., Yudovich, D., Belizaire, R., Puram, R.V., McConkey, M.E., et al. (2014). Generation of mouse models of myeloid malignancy with combinatorial genetic lesions using CRISPR-Cas9 genome editing. Nat Biotechnol 32(9), 941-946. doi: 10.1038/nbt.2951.

Hicks, D.A., Makova, N.Z., Gough, M., Parkin, E.T., Nalivaeva, N.N., and Turner, A.J. (2013). The amyloid precursor protein represses expression of acetylcholinesterase in neuronal cell lines. J Biol Chem 288(36), 26039-26051. doi: 10.1074/jbc.M113.461269.

Hoe, H.S., Fu, Z., Makarova, A., Lee, J.Y., Lu, C., Feng, L., et al. (2009a). The effects of amyloid precursor protein on postsynaptic composition and activity. J Biol Chem 284(13), 8495-8506. doi: 10.1074/jbc.M900141200.

Hoe, H.S., Lee, K.J., Carney, R.S., Lee, J., Markova, A., Lee, J.Y., et al. (2009b). Interaction of reelin with amyloid precursor protein promotes neurite outgrowth. J Neurosci 29(23), 7459-7473. doi: 10.1523/JNEUROSCI.4872-08.2009.

Hsieh, H., Boehm, J., Sato, C., Iwatsubo, T., Tomita, T., Sisodia, S., et al. (2006). AMPAR removal underlies Abeta-induced synaptic depression and dendritic spine loss. Neuron 52(5), 831-843. doi: 10.1016/j.neuron.2006.10.035.

Hu, Y., Hung, A.C., Cui, H., Dawkins, E., Bolos, M., Foa, L., et al. (2013). Role of cystatin C in amyloid precursor protein-induced proliferation of neural stem/progenitor cells. J Biol Chem 288(26), 18853-18862. doi: 10.1074/jbc.M112.443671.

Huysseune, S., Kienlen-Campard, P., and Octave, J.N. (2007). Fe65 does not stabilize AICD during activation of transcription in a luciferase assay. Biochem Biophys Res Commun 361(2), 317-322. doi: 10.1016/j.bbrc.2007.06.186.

Jinek, M., Chylinski, K., Fonfara, I., Hauer, M., Doudna, J.A., and Charpentier, E. (2012). A programmable dual-RNA-guided DNA endonuclease in adaptive bacterial immunity. Science 337(6096), 816-821. doi: $10.1126 /$ science. 1225829 .

Kamenetz, F., Tomita, T., Hsieh, H., Seabrook, G., Borchelt, D., Iwatsubo, T., et al. (2003). APP processing and synaptic function. Neuron 37(6), 925-937. 
838

839

840

841

842

843

844

845

846

847

848

849

850

851

852

853

854

855

856

857

858

859

860

861

862

863

864

865

866

867

868

869

870

871

872

873

874

875

876

877

878

879

880

881

882

883

884

885

886

887

Kanehisa, M., and Goto, S. (2000). KEGG: kyoto encyclopedia of genes and genomes. Nucleic Acids Res 28(1), 27-30.

Kimberly, W.T., Zheng, J.B., Town, T., Flavell, R.A., and Selkoe, D.J. (2005). Physiological regulation of the beta-amyloid precursor protein signaling domain by c-Jun N-terminal kinase JNK3 during neuronal differentiation. J Neurosci 25(23), 5533-5543. doi: 10.1523/JNEUROSCI.4883-04.2005.

Klevanski, M., Herrmann, U., Weyer, S.W., Fol, R., Cartier, N., Wolfer, D.P., et al. (2015). The APP Intracellular Domain Is Required for Normal Synaptic Morphology, Synaptic Plasticity, and Hippocampus-Dependent Behavior. $J$ Neurosci 35(49), 16018-16033. doi: 10.1523/JNEUROSCI.2009-15.2015.

Lassek, M., Weingarten, J., Wegner, M., Neupartl, M., Array, T.N., Harde, E., et al. (2017). APP Deletion Accounts for Age-Dependent Changes in the Bioenergetic Metabolism and in Hyperphosphorylated CaMKII at Stimulated Hippocampal Presynaptic Active Zones. Front Synaptic Neurosci 9, 1. doi: 10.3389/fnsyn.2017.00001.

Lee, K.J., Moussa, C.E., Lee, Y., Sung, Y., Howell, B.W., Turner, R.S., et al. (2010). Beta amyloidindependent role of amyloid precursor protein in generation and maintenance of dendritic spines. Neuroscience 169(1), 344-356. doi: 10.1016/j.neuroscience.2010.04.078.

Leslie, J.H., and Nedivi, E. (2011). Activity-regulated genes as mediators of neural circuit plasticity. Prog Neurobiol 94(3), 223-237. doi: 10.1016/j.pneurobio.2011.05.002.

Lin, Y., Bloodgood, B.L., Hauser, J.L., Lapan, A.D., Koon, A.C., Kim, T.K., et al. (2008). Activitydependent regulation of inhibitory synapse development by Npas4. Nature 455(7217), 1198-1204. doi: 10.1038 /nature07319.

Loebrich, S., and Nedivi, E. (2009). The function of activity-regulated genes in the nervous system. Physiol Rev 89(4), 1079-1103. doi: 10.1152/physrev.00013.2009.

Milosch, N., Tanriover, G., Kundu, A., Rami, A., Francois, J.C., Baumkotter, F., et al. (2014). Holo-APP and G-protein-mediated signaling are required for sAPPalpha-induced activation of the Akt survival pathway. Cell Death Dis 5, e1391. doi: 10.1038/cddis.2014.352.

Miyashita, A., Hatsuta, H., Kikuchi, M., Nakaya, A., Saito, Y., Tsukie, T., et al. (2014). Genes associated with the progression of neurofibrillary tangles in Alzheimer's disease. Transl Psychiatry 4, e396. doi: $10.1038 /$ tp.2014.35.

Muller, U., Cristina, N., Li, Z.W., Wolfer, D.P., Lipp, H.P., Rulicke, T., et al. (1994). Behavioral and anatomical deficits in mice homozygous for a modified beta-amyloid precursor protein gene. Cell 79(5), 755-765.

Muller, U.C., Deller, T., and Korte, M. (2017). Not just amyloid: physiological functions of the amyloid precursor protein family. Nat Rev Neurosci 18(5), 281-298. doi: 10.1038/nrn.2017.29.

Muller, U.C., Pietrzik, C.U., and Deller, T. (2012). The physiological functions of the beta-amyloid precursor protein APP. Exp Brain Res 217(3-4), 325-329. doi: 10.1007/s00221-012-3039-2.

Muller, U.C., and Zheng, H. (2012). Physiological functions of APP family proteins. Cold Spring Harb Perspect Med 2(2), a006288. doi: 10.1101/cshperspect.a006288.

Pardossi-Piquard, R., and Checler, F. (2012). The physiology of the beta-amyloid precursor protein intracellular domain AICD. J Neurochem 120 Suppl 1, 109-124. doi: 10.1111/j.14714159.2011.07475.x.

Pickering, M., Pickering, B.W., Murphy, K.J., and O'Connor, J.J. (2008). Discrimination of cell types in mixed cortical culture using calcium imaging: a comparison to immunocytochemical labeling. $J$ Neurosci Methods 173(1), 27-33. doi: 10.1016/j.jneumeth.2008.05.014.

Pierrot, N., Tyteca, D., D'Auria, L., Dewachter, I., Gailly, P., Hendrickx, A., et al. (2013). Amyloid precursor protein controls cholesterol turnover needed for neuronal activity. EMBO Mol Med 5(4), 608-625. doi: 10.1002/emmm.201202215.

Priller, C., Bauer, T., Mitteregger, G., Krebs, B., Kretzschmar, H.A., and Herms, J. (2006). Synapse formation and function is modulated by the amyloid precursor protein. $J$ Neurosci 26(27), 72127221. doi: 10.1523/JNEUROSCI.1450-06.2006. 
888

889

890

891

892

893

894

895

896

897

898

899

900

901

902

903

904

905

906

907

908

909

910

911

912

913

914

915

916

917

918

919

920

921

922

923

924

925

926

927

928

929

930

931

932

933

934

935

936

937
Puzzo, D., Privitera, L., Fa, M., Staniszewski, A., Hashimoto, G., Aziz, F., et al. (2011). Endogenous amyloid-beta is necessary for hippocampal synaptic plasticity and memory. Ann Neurol 69(5), 819830. doi: 10.1002/ana.22313.

Restituito, S., Khatri, L., Ninan, I., Mathews, P.M., Liu, X., Weinberg, R.J., et al. (2011). Synaptic autoregulation by metalloproteases and gamma-secretase. J Neurosci 31(34), 12083-12093. doi: 10.1523/JNEUROSCI.2513-11.2011.

Ring, S., Weyer, S.W., Kilian, S.B., Waldron, E., Pietrzik, C.U., Filippov, M.A., et al. (2007). The secreted beta-amyloid precursor protein ectodomain APPs alpha is sufficient to rescue the anatomical, behavioral, and electrophysiological abnormalities of APP-deficient mice. J Neurosci 27(29), 7817 7826. doi: 10.1523/JNEUROSCI.1026-07.2007.

Ritchie, M.E., Phipson, B., Wu, D., Hu, Y., Law, C.W., Shi, W., et al. (2015). limma powers differential expression analyses for RNA-sequencing and microarray studies. Nucleic Acids Res 43(7), e47. doi: 10.1093/nar/gkv007.

Santos, S.F., Pierrot, N., Morel, N., Gailly, P., Sindic, C., and Octave, J.N. (2009). Expression of human amyloid precursor protein in rat cortical neurons inhibits calcium oscillations. J Neurosci 29(15), 4708-4718. doi: 10.1523/JNEUROSCI.4917-08.2009.

Seabrook, G.R., Smith, D.W., Bowery, B.J., Easter, A., Reynolds, T., Fitzjohn, S.M., et al. (1999). Mechanisms contributing to the deficits in hippocampal synaptic plasticity in mice lacking amyloid precursor protein. Neuropharmacology 38(3), 349-359.

Senechal, Y., Kelly, P.H., Cryan, J.F., Natt, F., and Dev, K.K. (2007). Amyloid precursor protein knockdown by siRNA impairs spontaneous alternation in adult mice. J Neurochem 102(6), 19281940. doi: 10.1111/j.1471-4159.2007.04672.x.

Shariati, S.A., and De Strooper, B. (2013). Redundancy and divergence in the amyloid precursor protein family. FEBS Lett 587(13), 2036-2045. doi: 10.1016/j.febslet.2013.05.026.

Sim, S., Antolin, S., Lin, C.W., Lin, Y., and Lois, C. (2013). Increased cell-intrinsic excitability induces synaptic changes in new neurons in the adult dentate gyrus that require Npas4. J Neurosci 33(18), 7928-7940. doi: 10.1523/JNEUROSCI.1571-12.2013.

Snyder, E.M., Nong, Y., Almeida, C.G., Paul, S., Moran, T., Choi, E.Y., et al. (2005). Regulation of NMDA receptor trafficking by amyloid-beta. Nat Neurosci 8(8), 1051-1058. doi: 10.1038/nn1503.

Spiegel, I., Mardinly, A.R., Gabel, H.W., Bazinet, J.E., Couch, C.H., Tzeng, C.P., et al. (2014). Npas4 regulates excitatory-inhibitory balance within neural circuits through cell-type-specific gene programs. Cell 157(5), 1216-1229. doi: 10.1016/j.cell.2014.03.058.

Stanga, S., Zanou, N., Audouard, E., Tasiaux, B., Contino, S., Vandermeulen, G., et al. (2016). APPdependent glial cell line-derived neurotrophic factor gene expression drives neuromuscular junction formation. FASEB J 30(5), 1696-1711. doi: 10.1096/fj.15-278739.

Sun, X., and Lin, Y. (2016). Npas4: Linking Neuronal Activity to Memory. Trends Neurosci 39(4), 264275. doi: 10.1016/j.tins.2016.02.003.

Tyan, S.H., Shih, A.Y., Walsh, J.J., Maruyama, H., Sarsoza, F., Ku, L., et al. (2012). Amyloid precursor protein (APP) regulates synaptic structure and function. Mol Cell Neurosci 51(1-2), 43-52. doi: 10.1016/j.mcn.2012.07.009.

Waldron, E., Isbert, S., Kern, A., Jaeger, S., Martin, A.M., Hebert, S.S., et al. (2008). Increased AICD generation does not result in increased nuclear translocation or activation of target gene transcription. Exp Cell Res 314(13), 2419-2433. doi: 10.1016/j.yexcr.2008.05.003.

West, A.E., and Greenberg, M.E. (2011). Neuronal activity-regulated gene transcription in synapse development and cognitive function. Cold Spring Harb Perspect Biol 3(6). doi: 10.1101/cshperspect.a005744.

Weyer, S.W., Zagrebelsky, M., Herrmann, U., Hick, M., Ganss, L., Gobbert, J., et al. (2014). Comparative analysis of single and combined APP/APLP knockouts reveals reduced spine density in APP-KO mice that is prevented by APPsalpha expression. Acta Neuropathol Commun 2, 36. doi: $10.1186 / 2051-5960-2-36$. 
938

939

940

941

942

943

944

945

946

947

948

949

950

951

952

953
White, A.R., Zheng, H., Galatis, D., Maher, F., Hesse, L., Multhaup, G., et al. (1998). Survival of cultured neurons from amyloid precursor protein knock-out mice against Alzheimer's amyloid-beta toxicity and oxidative stress. J Neurosci 18(16), 6207-6217.

Wu, D., Lim, E., Vaillant, F., Asselin-Labat, M.L., Visvader, J.E., and Smyth, G.K. (2010). ROAST: rotation gene set tests for complex microarray experiments. Bioinformatics 26(17), 2176-2182. doi: 10.1093/bioinformatics/btq401.

Young-Pearse, T.L., Bai, J., Chang, R., Zheng, J.B., LoTurco, J.J., and Selkoe, D.J. (2007). A critical function for beta-amyloid precursor protein in neuronal migration revealed by in utero RNA interference. J Neurosci 27(52), 14459-14469. doi: 10.1523/JNEUROSCI.4701-07.2007.

Zheng, H., Jiang, M., Trumbauer, M.E., Sirinathsinghji, D.J., Hopkins, R., Smith, D.W., et al. (1995). betaAmyloid precursor protein-deficient mice show reactive gliosis and decreased locomotor activity. Cell 81(4), 525-531.

Zou, C., Crux, S., Marinesco, S., Montagna, E., Sgobio, C., Shi, Y., et al. (2016). Amyloid precursor protein maintains constitutive and adaptive plasticity of dendritic spines in adult brain by regulating Dserine homeostasis. EMBO J 35(20), 2213-2222. doi: 10.15252/embj.201694085. 
Figure 1

A

Up- and down-regulated genes

\begin{tabular}{|c|cccccc|}
\hline $\begin{array}{c}\text { Linear } \\
\text { fold } \\
\text { change }\end{array}$ & \multicolumn{2}{|c}{ E18 } & \multicolumn{2}{c}{ DIV3 } & \multicolumn{2}{c|}{ DIV7 } \\
\hline$\geq 1,25$ & 90 & Down & Up & Down & Up & Down \\
$\geq 1,5$ & 16 & 4 & 93 & 54 & 73 & 56 \\
$\geq 2$ & 4 & 1 & 2 & 3 & 0 & 2 \\
\hline
\end{tabular}

C

\begin{tabular}{|c|c|c|}
\hline DIV7 & $\begin{array}{c}\text { Gene } \\
\text { symbol }\end{array}$ & $\begin{array}{c}\text { Fold change } \\
*\end{array}$ \\
\hline \multirow{10}{*}{ 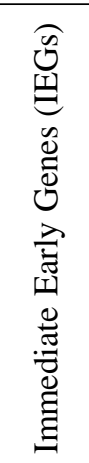 } & $A p p$ & 0,05 \\
\hline & Ier2 & 0,52 \\
\hline & $A r c$ & 0,56 \\
\hline & Npas4 & 0,57 \\
\hline & Fosb & 0,57 \\
\hline & Fos & 0,60 \\
\hline & Egrl & 0,66 \\
\hline & Egr2 & 0,74 \\
\hline & Egr3 & 0,77 \\
\hline & Egr4 & 0,78 \\
\hline
\end{tabular}

* linear fold change (APP-/- vs. APP+/+)

\section{B}

\section{KEGG Pathways}

Nb. Genes Direction

\begin{tabular}{|c|c|c|}
\hline E18 & & \\
\hline Base excision repair & 32 & Up \\
\hline p53 signaling pathway & 70 & Up \\
\hline Terpenoid backbone biosynthesis & 14 & Down \\
\hline Small cell lung cancer & 88 & Up \\
\hline Pyrimidine metabolism & 96 & Up \\
\hline DIV3 & & \\
\hline Proteasome & 42 & Up \\
\hline $\begin{array}{l}\text { RIG-I-like receptor signaling } \\
\text { pathway }\end{array}$ & 67 & Down \\
\hline Terpenoid backbone biosynthesis & 14 & Up \\
\hline Glycosaminoglycan biosynthesis & 14 & Up \\
\hline Steroid biosynthesis & 17 & Up \\
\hline DIV7 & & \\
\hline Homologous recombination & 26 & Down \\
\hline $\begin{array}{l}\text { Glycosylphosphatidylinositol(GPI } \\
\text { )-anchor biosynthesis }\end{array}$ & 23 & Down \\
\hline $\begin{array}{l}\text { Glycosaminoglycan biosynthesis - } \\
\text { keratan sulfate }\end{array}$ & 14 & Up \\
\hline ECM-receptor interaction & 83 & Down \\
\hline Long-term potentiation & 64 & Up \\
\hline
\end{tabular}

D

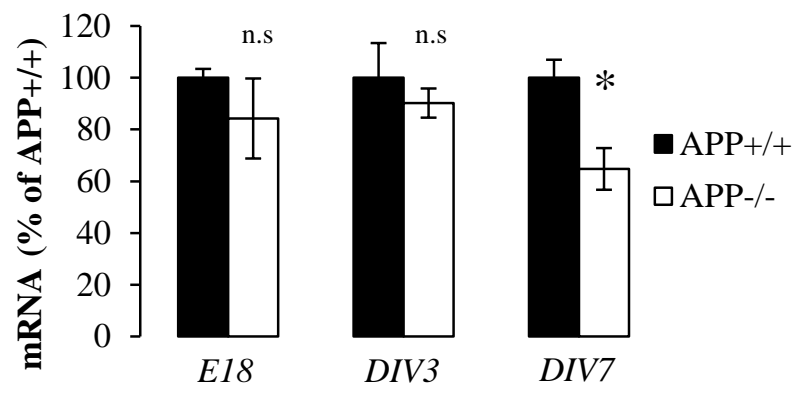


Figure 2

A

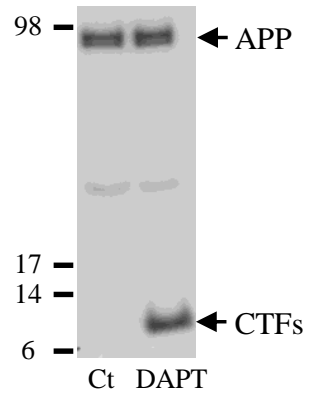

C

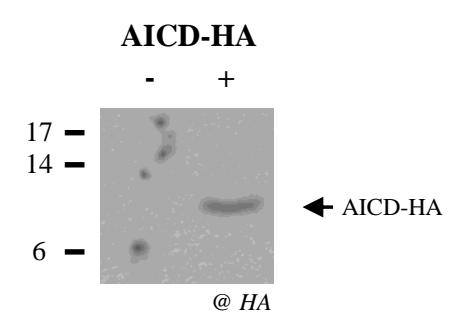

E

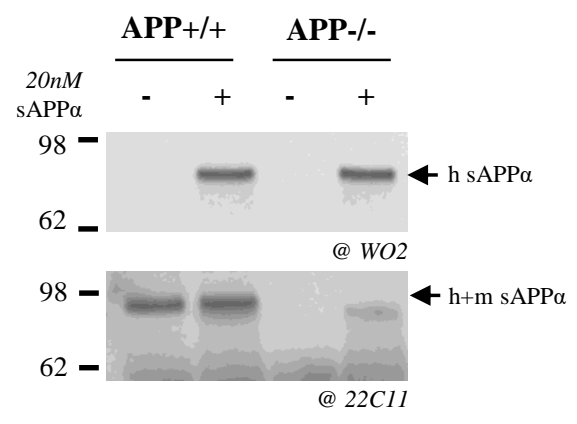

B

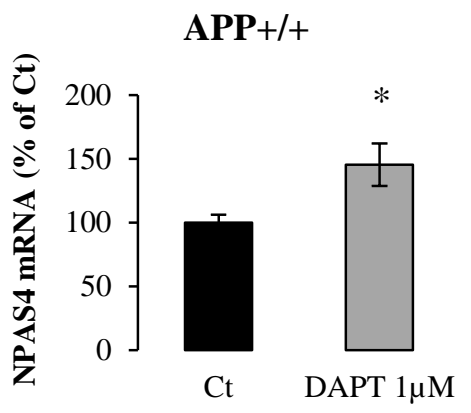

D

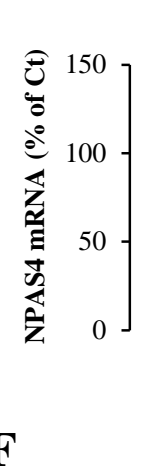

APP+/+
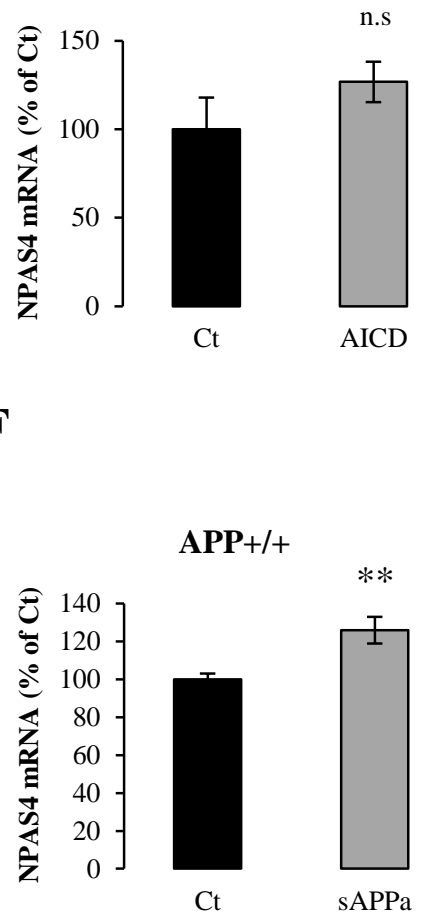

APP-/-

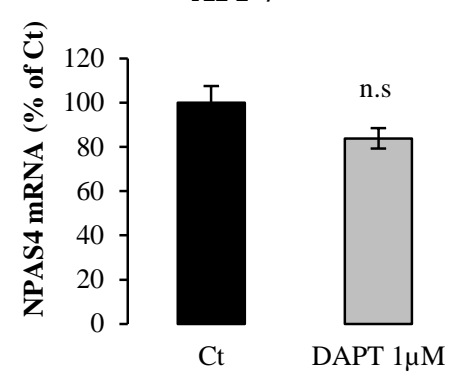

$\mathrm{Ct}$

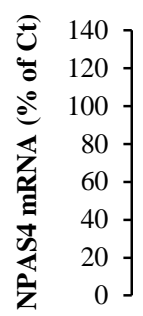

APP-/-
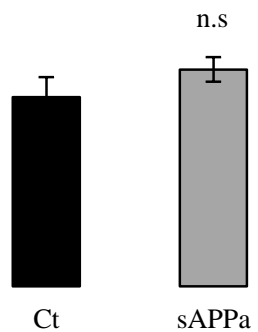
Figure 3

A

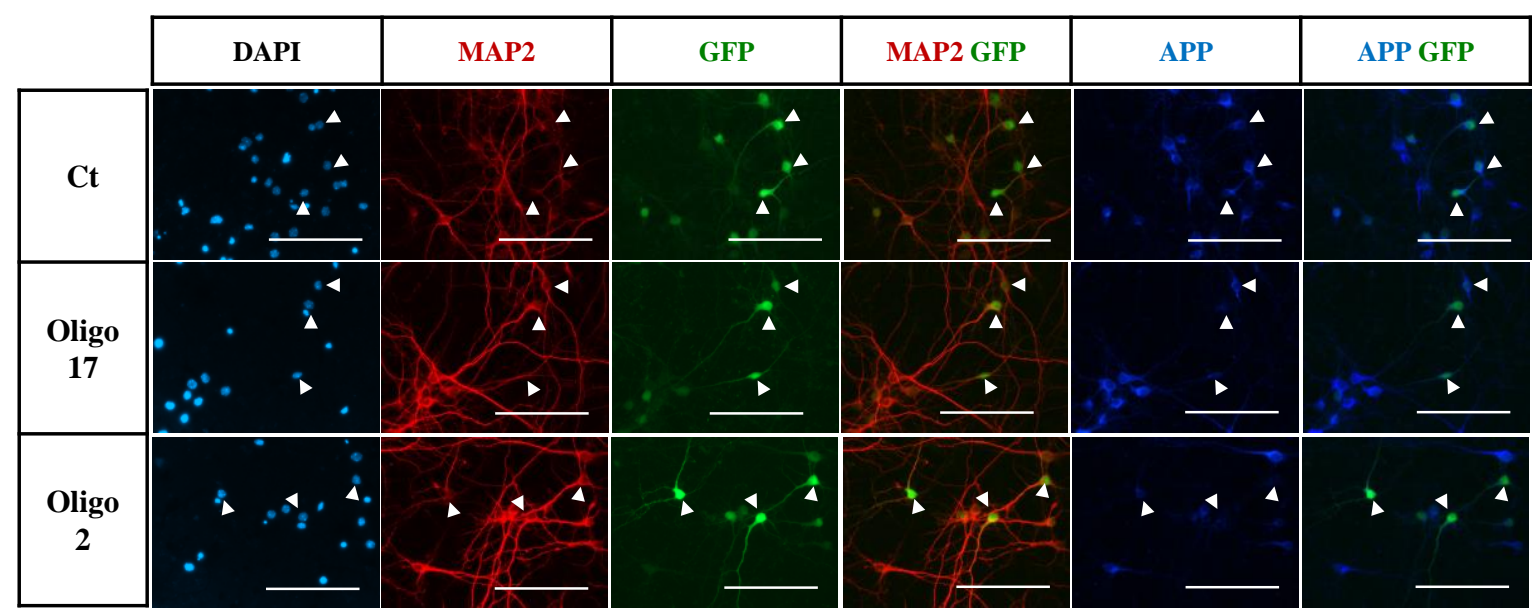

B

C

APP signal intensity in GFP-positive neurons

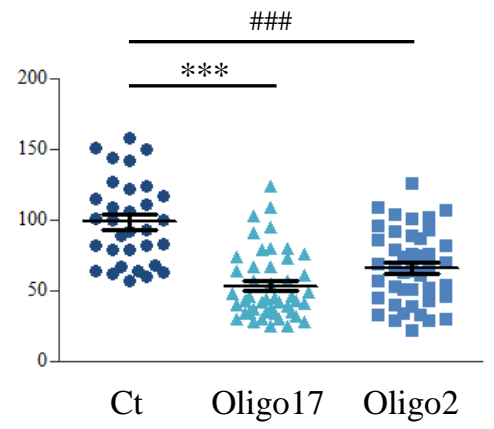

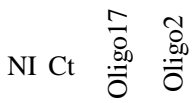

D

$\underline{\text { Non-infected neurons }}$
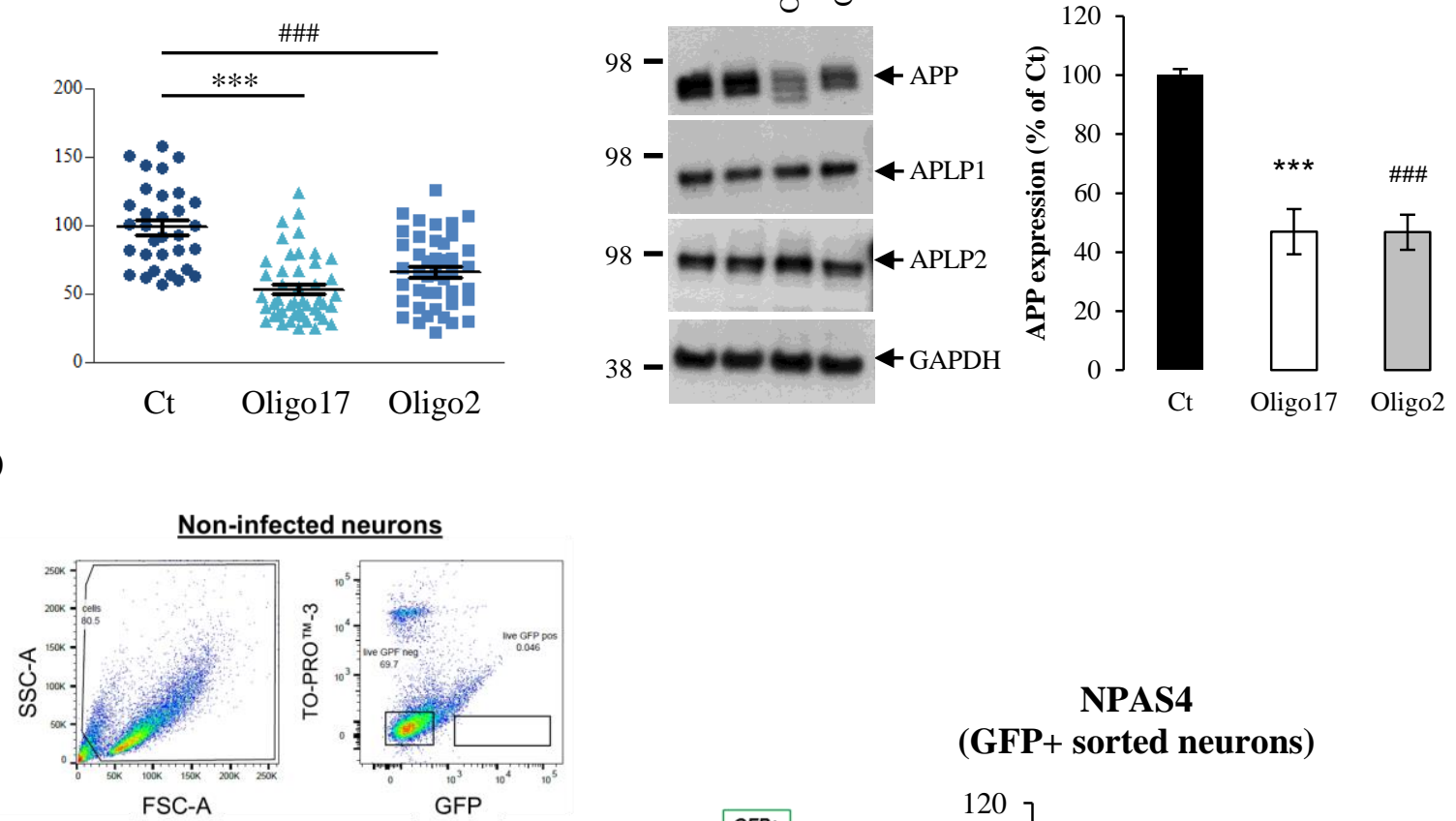

GFP-infected neurons
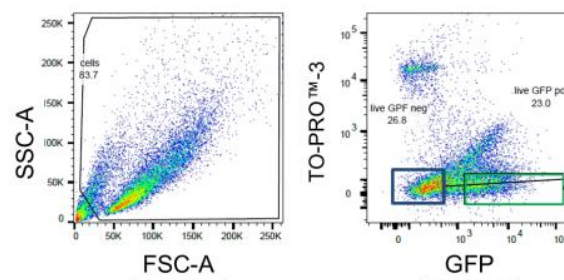

GFP

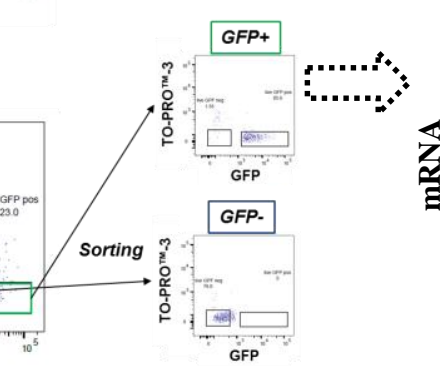

\section{NPAS4 \\ (GFP+ sorted neurons)}

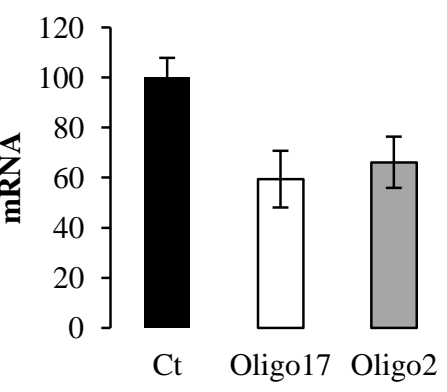


Figure 4

A

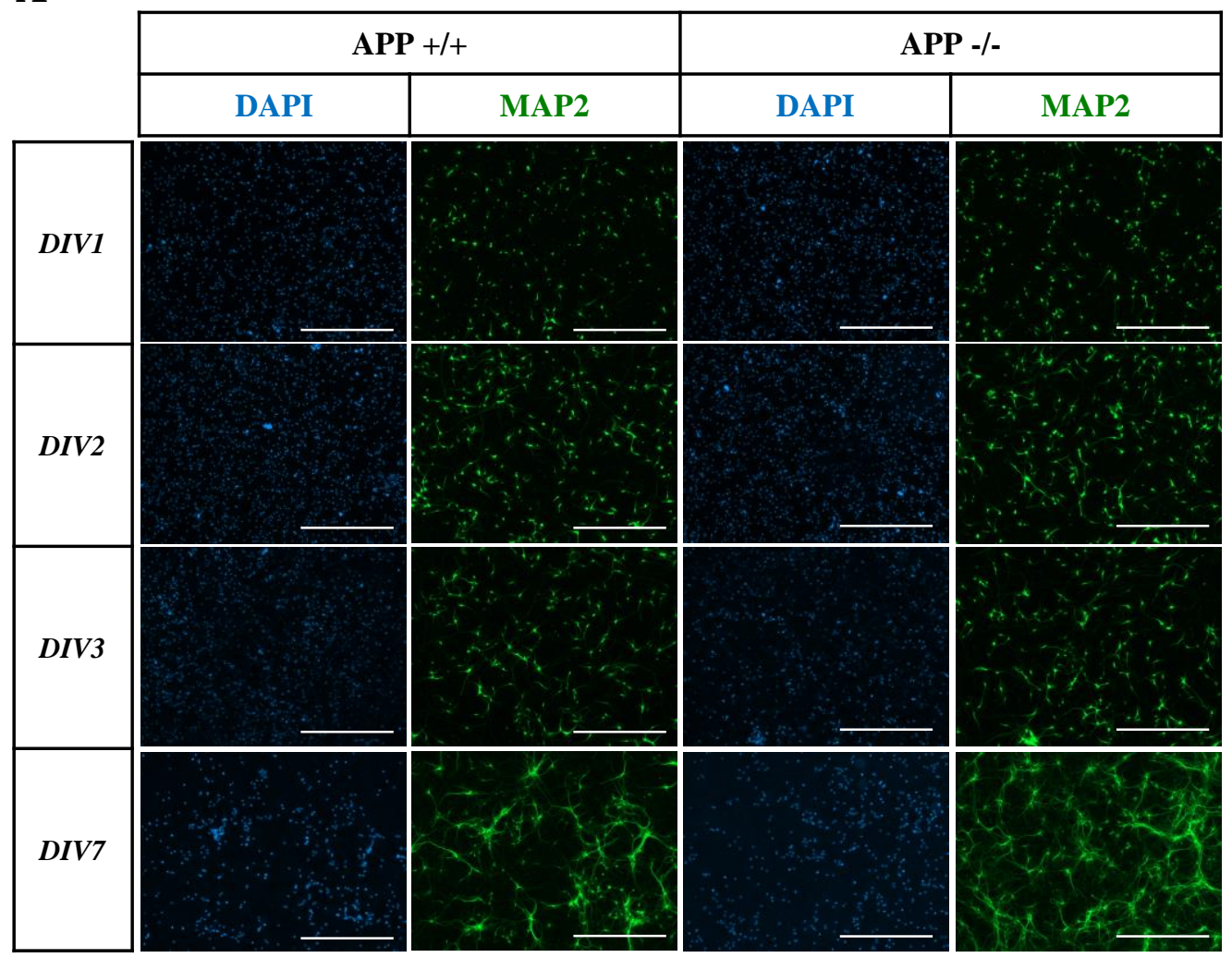

B

MAP2 area/cell number (\% of $\mathrm{APP}+/+$ )

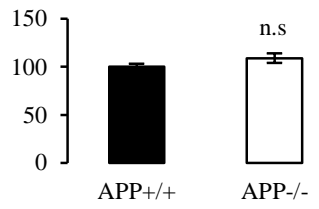

$\left.\begin{array}{r}150 \\ 100 \\ 50 \\ 0\end{array}\right] \underset{\mathrm{APP}+/+}{\underbrace{\mathrm{n} . \mathrm{s}}_{\mathrm{APP}-/-}}$

$\left.\begin{array}{r}150 \\ 100 \\ 50 \\ 0\end{array}\right] \underset{\mathrm{APP}+/+}{{ }_{\mathrm{APP}-/-}^{\mathrm{n} . \mathrm{s}}}$

$\left.\begin{array}{r}150 \\ 100 \\ 50 \\ 0\end{array}\right] \underset{\mathrm{APP}+/+}{{ }_{\mathrm{APP}-/-}^{*}}$ 


\section{Figure 5}

A

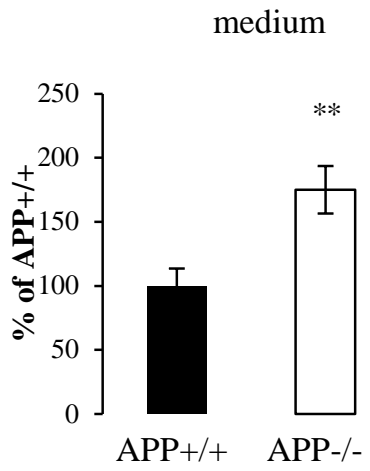

GABA

cell

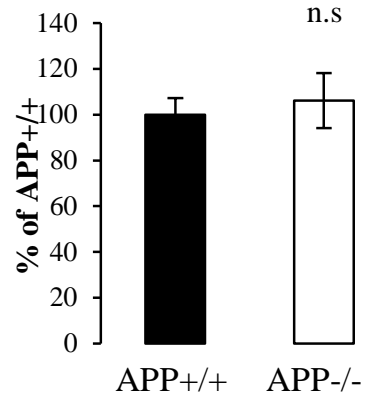

B

Glutamate

medium

cell

n.s

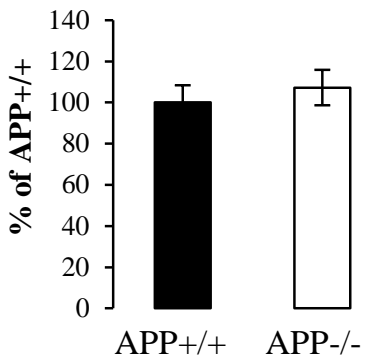

n.s

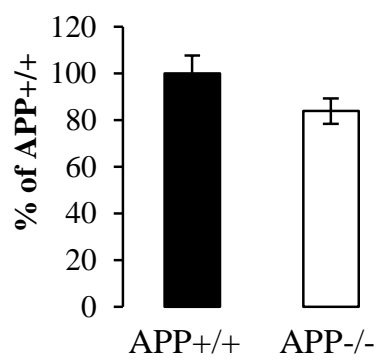

C

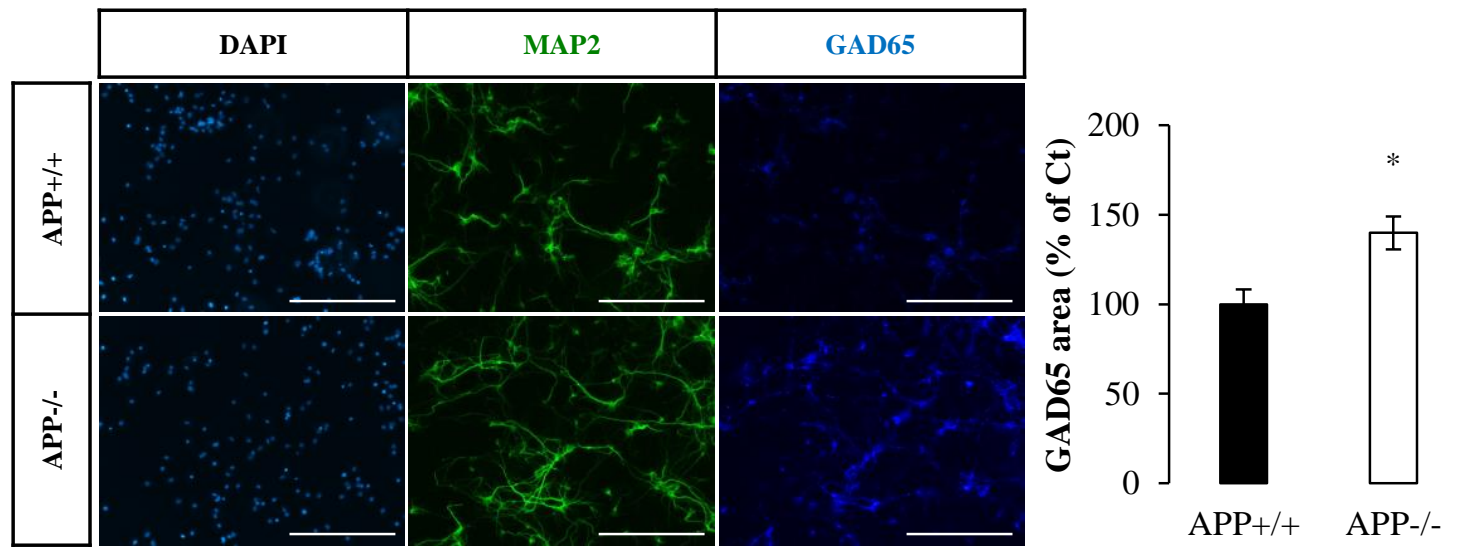

D

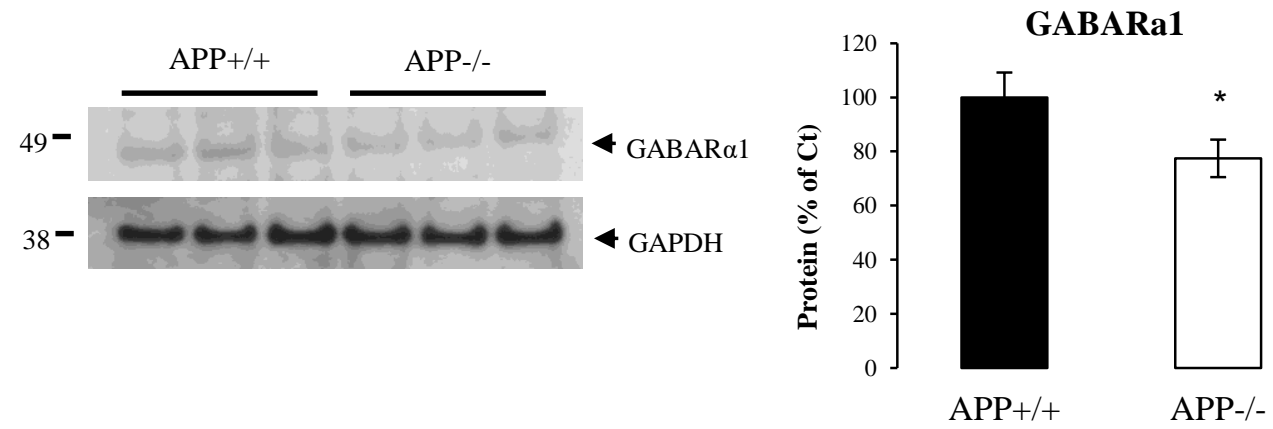




\section{Figure 6}

A

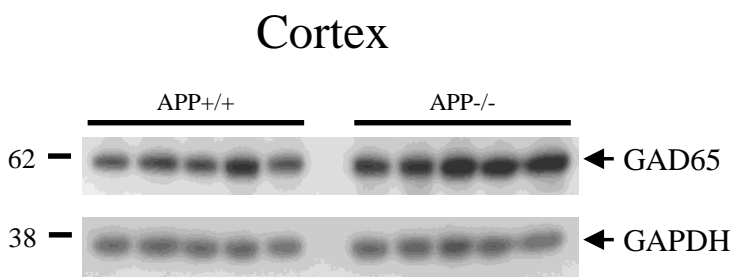

B

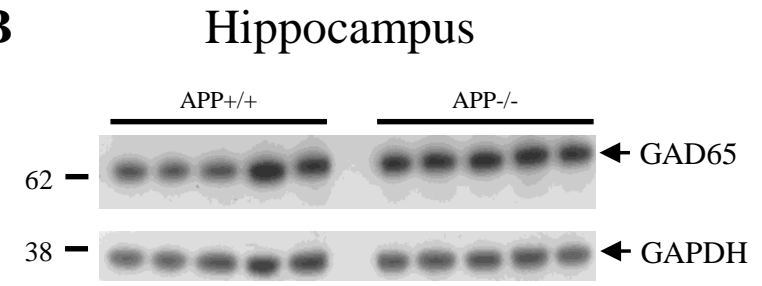

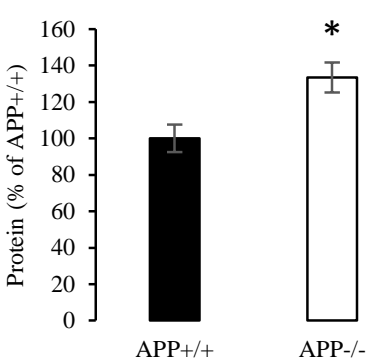

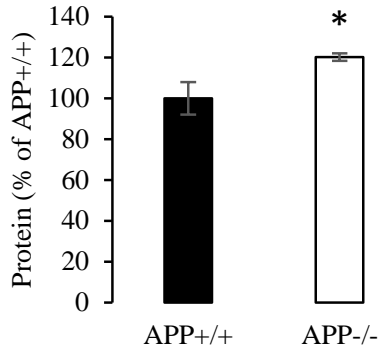


Figure 7

A

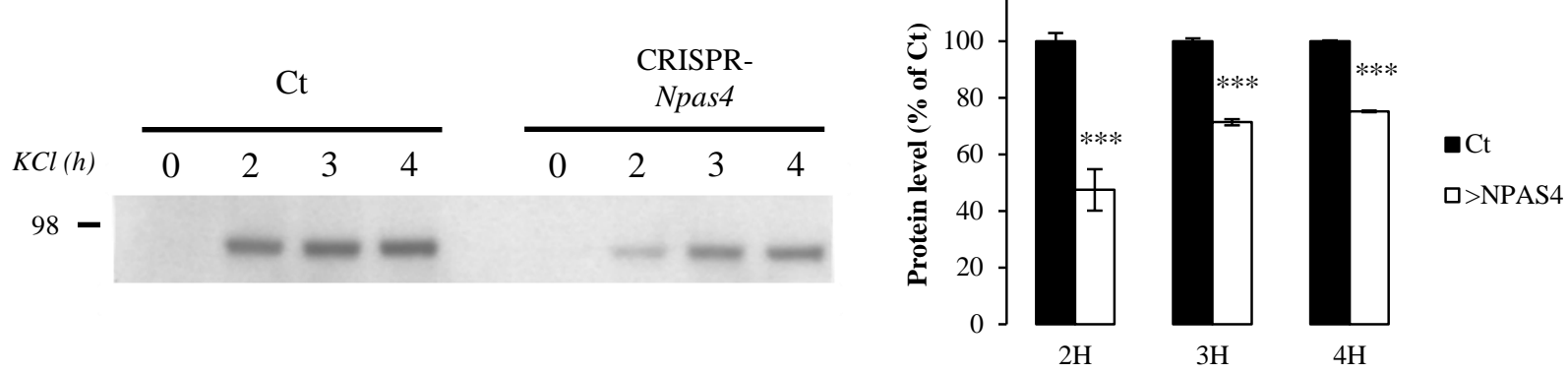

B
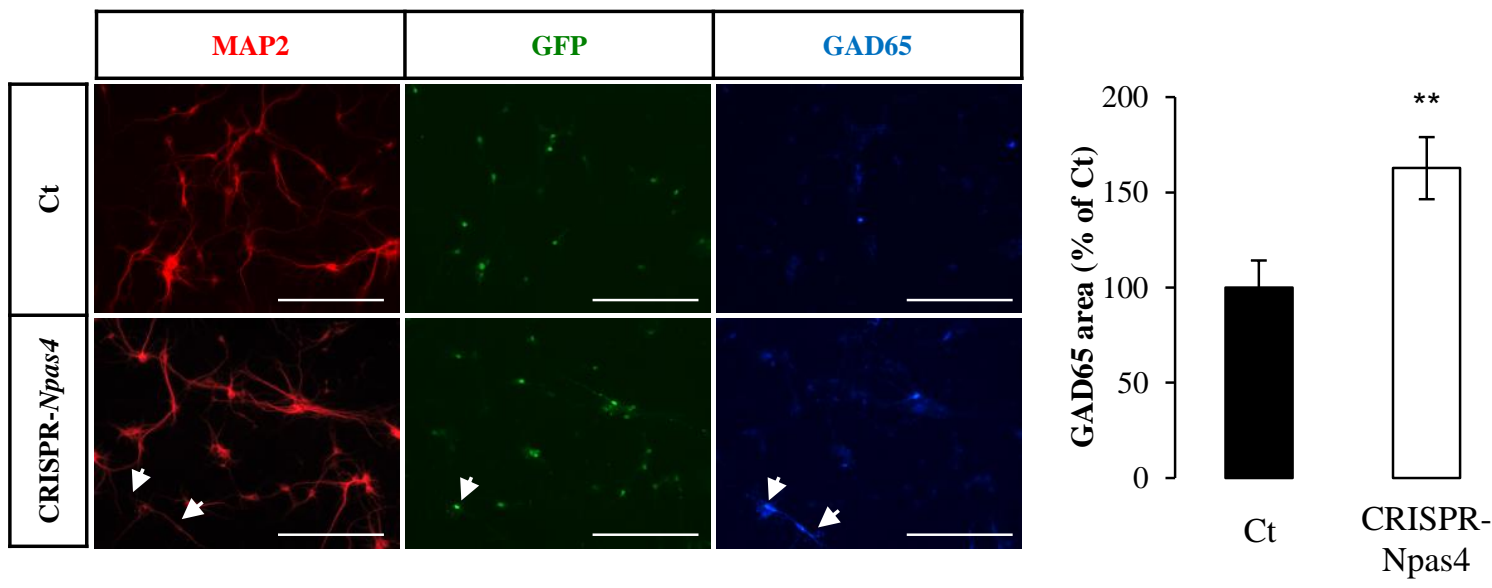

C

medium

D
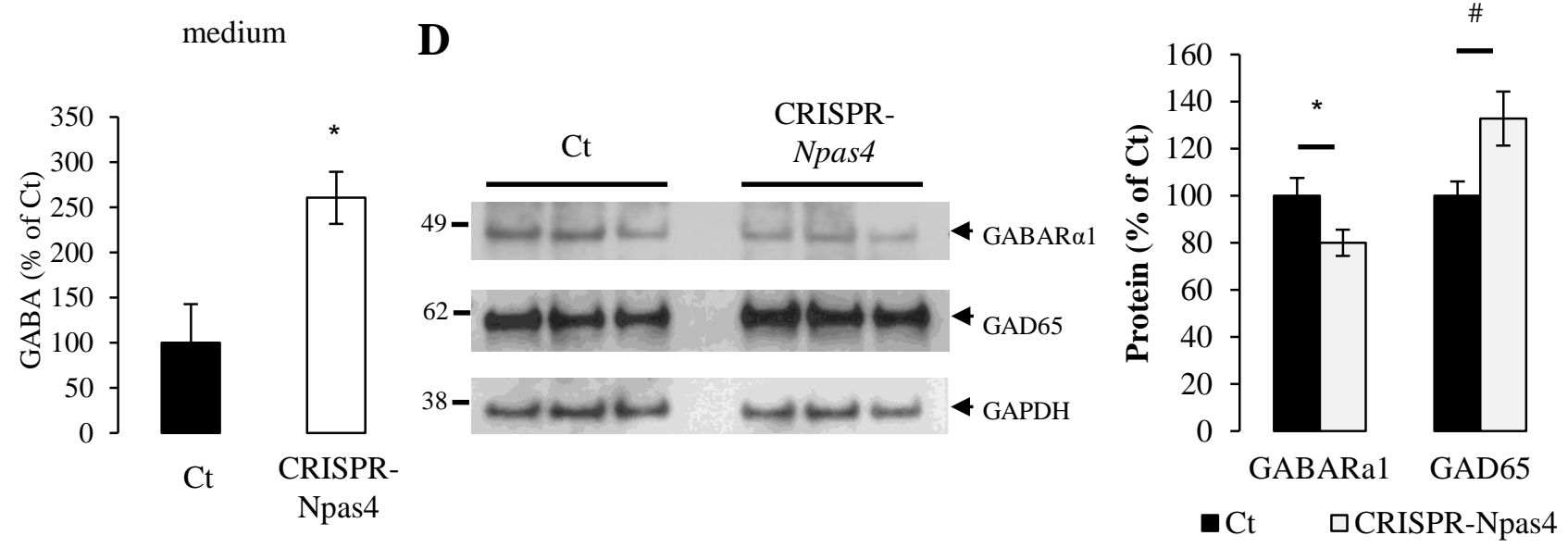


\section{Supplemental Figure 1: Experimental Workflow and model characterization}

A

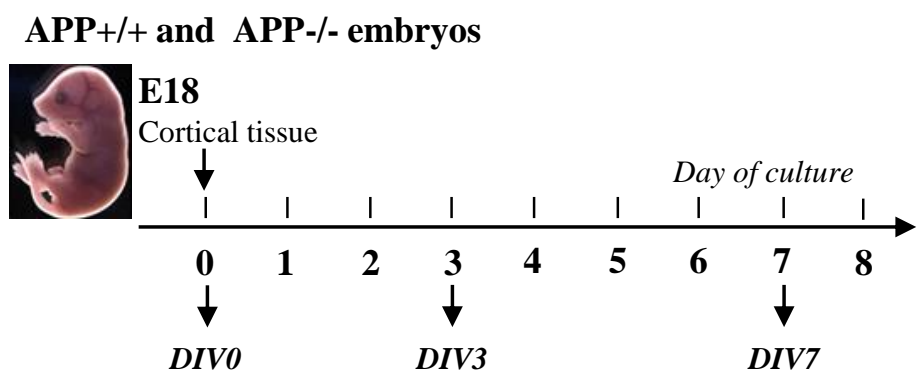

GeneChip ${ }^{\circledR}$ Mouse Transcriptome Array 1.0 Affymetrix

B

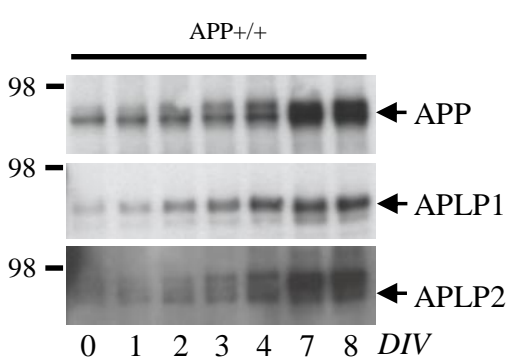

C

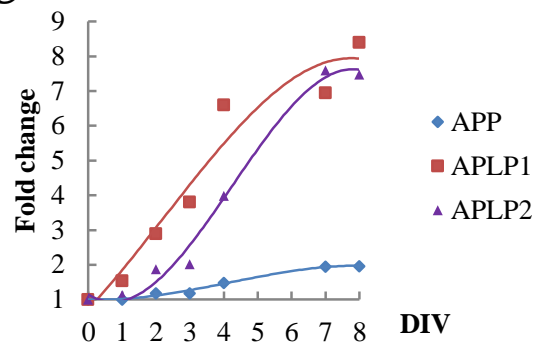

D

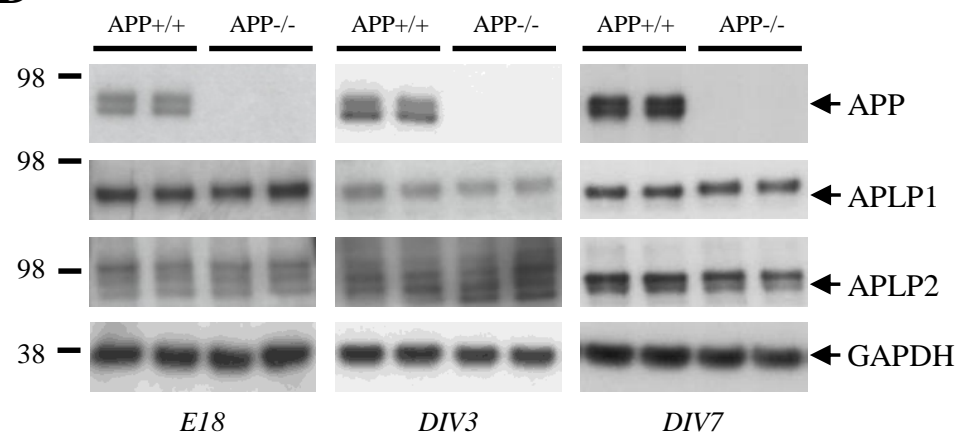

$\mathbf{E}$

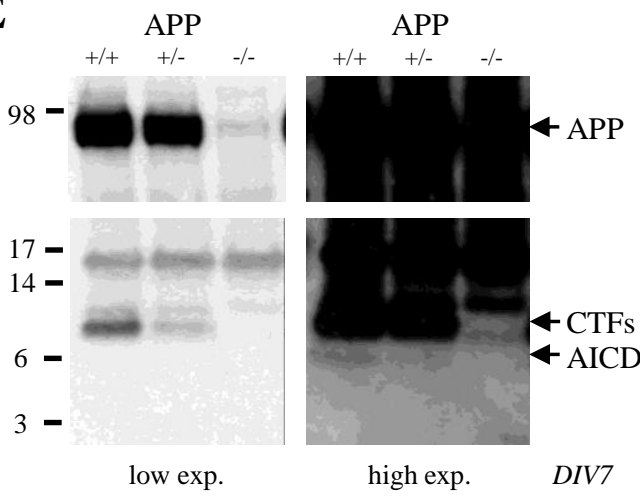

Figure S1: Experimental workflow and model characterization

A) Experimental design used for the study. Cortical tissue was taken at embryonic day 18 (E18); neurons were cultured and experiments were mainly carried out after 3 and 7 days in vitro (DIV3 and DIV7). Transcriptome analysis was performed on embryonic cortex (E18) and at DIV3 or DIV7. B) APP, APLP1 and APLP2 expressions were analyzed by Western blotting at the indicated days of culture in APP+/+ neurons. C) Quantification of APP, APLP1 and APLP2 expression over time in APP+/+ neurons. Accumulation is represented as fold change over the signal measured at day 0. Quantification was performed from one neuronal culture D) APLP1 and APLP2 expressions are not modified in cortical tissue at E18 and primary neuron cultures at DIV3 and DIV 7. Expression of APP, APLP1, APLP2 was analyzed by Western blotting of cells lysates from APP+/+ and APP-/- primary neuron cultures. E) Samples from primary cultures at DIV7 (APP+/+, APP+/- and APP-/- neurons) were probed (Western blotting) with an antibody directed against APP Cterminus for APP C-terminal fragments (CTFs) and AICD. Low and high exposures of a typical blot are shown. Arrows indicate the expected position of APP holoprotein, APP CTFs and AICD. 


\section{Supplemental Figure 2 : Egr1 and Egr3 expressions are not modified in APP deficient neurons}

A

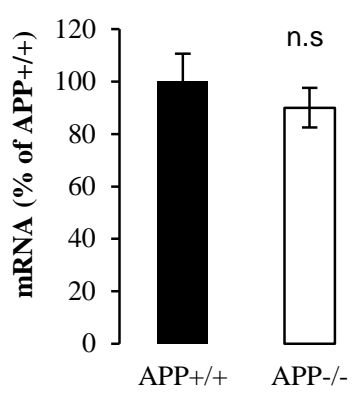

B<smiles>[Mg][Mg]</smiles>

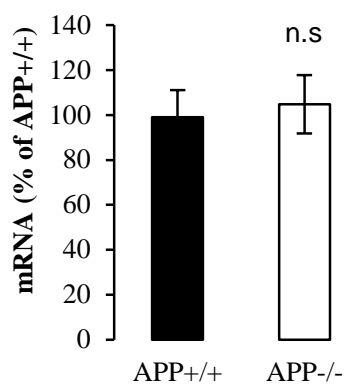

Figure S2: Egr1 and Egr3 expressions are not modified in APP deficient neurons

Egr1 and Egr3 expressions were evaluated in APP+/+ vs. APP-/- primary neurons at DIV7. A) Egr1 mRNA level was measured by qPCR $(n=6, N=3)$ at DIV7. Results (mean \pm SEM) are given as percentage of controls $(A P P+/+) n \cdot s=$ nonsignificant, Student's t-test. B) Egr3 mRNA level was measured by qPCR ( $\mathrm{n}=6, \mathrm{~N}=3$ ) at DIV7. Results (mean $\pm \mathrm{SEM})$ are given as percentage of controls $(\mathrm{APP}+/+) \mathrm{n} . \mathrm{s}=$ non-significant, Student's t-test. 


\section{Supplemental Figure 3: Astrocytes and Npas4 expression}

A

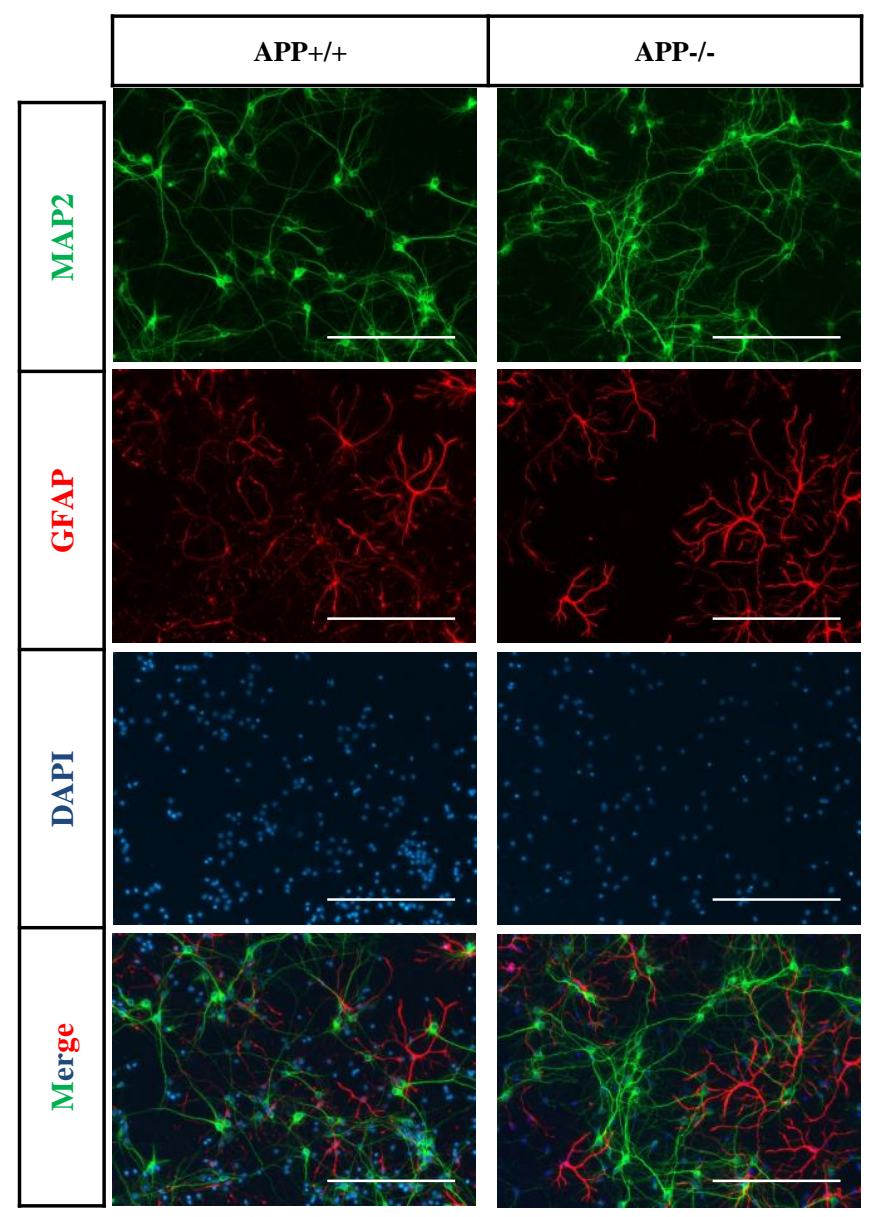

B

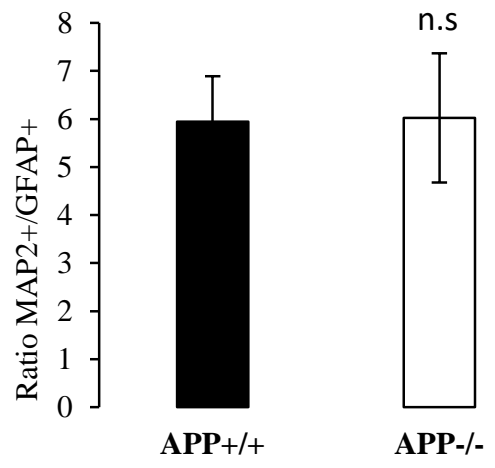

C

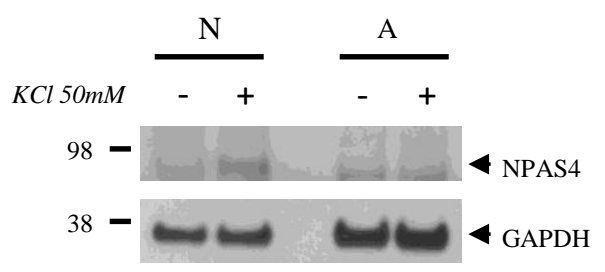

Figure S3: Astrocytes in primary neuron culture and their implication in Npas4 expression.

A) Primary culture of cortical neurons at DIV7. Cultures were immunostained with the neuron specific protein MAP2 (green), the glial specific protein GFAP (red) and the DAPI (light blue). Scale bar $=400 \mu \mathrm{m}$. B) Quantification of neurons (MAP2+) and astrocytes (GFAP+) in the primary cortical culture. At least five fields per coverslip were analyzed for $\mathrm{APP}+/+$ and APP-/- cultures in two independent experiments $(\mathrm{n} \geq 5, \mathrm{~N}=2)$. Results are expressed as the ratio of MAP2+ (neurons) and GFAP+ (astrocytes) (mean \pm s.e.m). n.s= non-significant, Mann-Whitney test. C) Western blotting analysis of Npas4 induction in neurons $(\mathrm{N})$ and astrocytes (A) after depolarization with $50 \mathrm{mM}$ potassium chloride $(\mathrm{KCl})$ for 2 hours. 


\section{Supplemental Figure 4: Infectivity and toxicity of lentiviral CRISPR-Cas9 vectors}

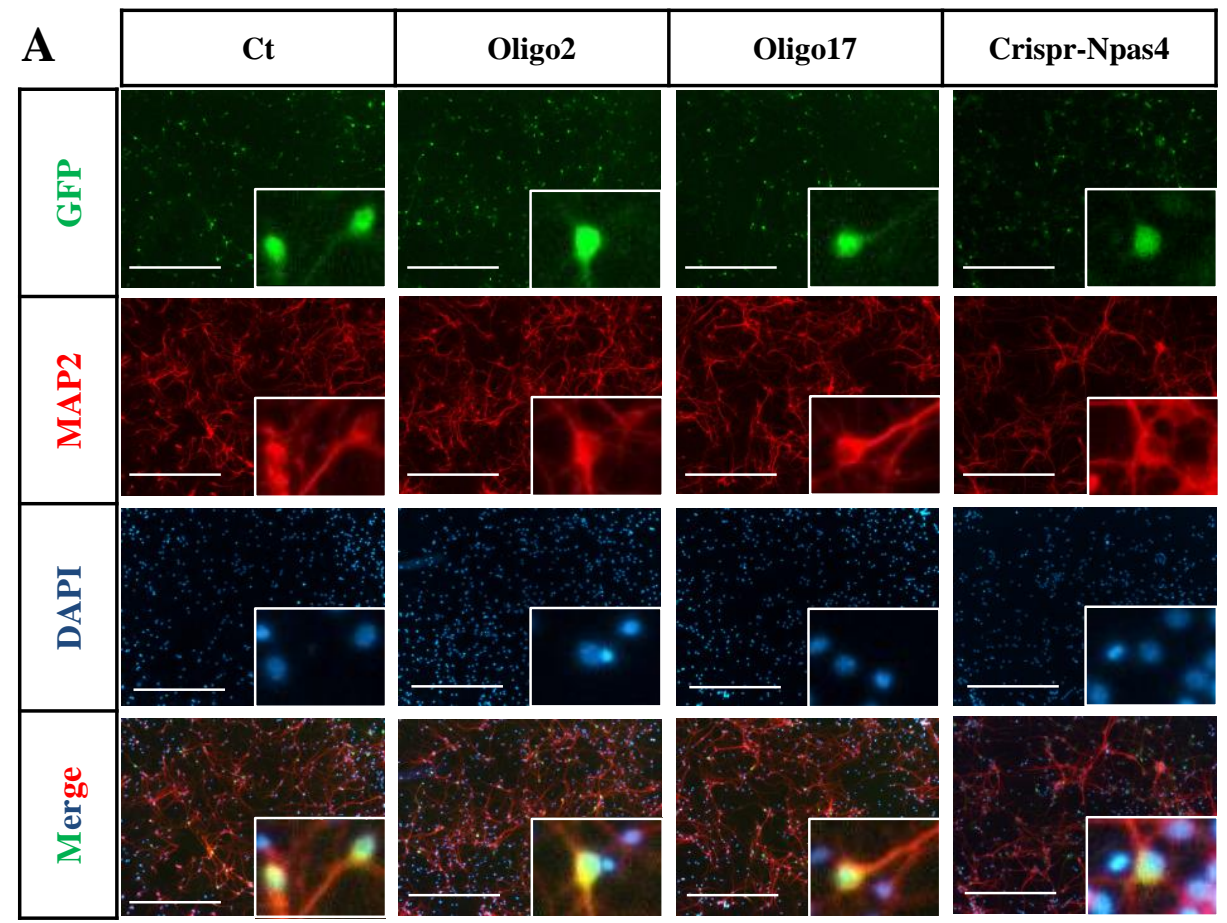

B

C

Infectivity of CRISPR/Cas9 lentiviral vectors

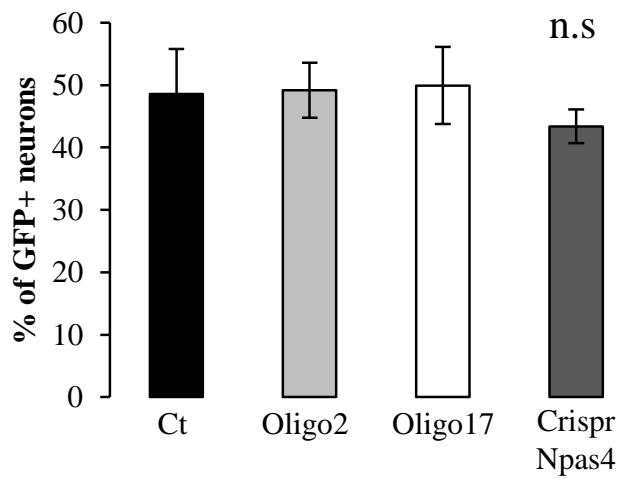

Toxicity of CRISPR-Cas9 lentiviral vectors
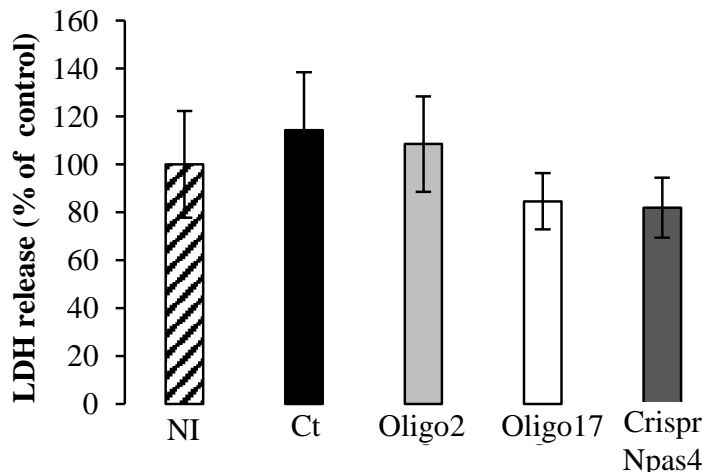

\section{Figure S4: Infectivity and toxicity of lentiviral CRISPR-Cas9 vectors}

A) Cortical neurons were infected at DIV1 with lentiviruses expressing sgRNAs (Oligo2, Oligo17 or CRISPR-Npas4) or no sgRNA (Ct), SpCas9 and GFP. Cultures were immunostained for MAP2 (red) and DAPI (light blue) at DIV7. Scale bar $=400 \mu \mathrm{m}$. B) Quantification of GFP+ neurons (GFP+/MAP2+) in total neuron population (MAP2+) after lentiviral CRISPR-Cas9 infection with control (Ct), Oligo2, Oligo17 or CRISPR-Npas4. At least five fields were analyzed for each lentiviral vector in two independent experiments $(\mathrm{n} \geq 5, \mathrm{~N}=2)$. Results are expressed as percentage of GFP+/MAP2+ cells in total MAP2+ cells (mean \pm s.e.m). n.s= non-significant, Kruskal-Wallis test and Dunn's multiple comparison test. C) Measurement of LDH activity released after infection (DIV7) of primary neuron with control (Ct), Oligo2, Oligo17 or CRISPR-Npas4 at DIV7 lentiviral vectors. Background LDH release was determined in non-infected control cultures (NI). Results were expressed as percentage of total LDH release measured in non-infected control cultures (NI) in 2 independent experiments $(\mathrm{n}=12, \mathrm{~N}=2)$. 


\section{Supplemental Figure 5: Weak modification of glutamate responses in APP-/- neurons}

A

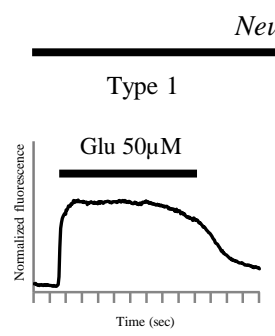

Neuronal

Type 2

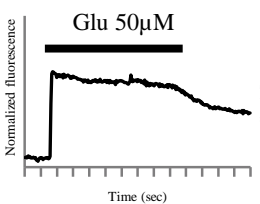

Time (sec)
Non-neuronal

Type 3

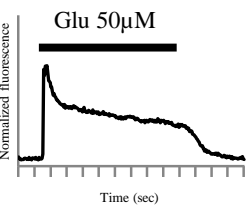

ime (sec)

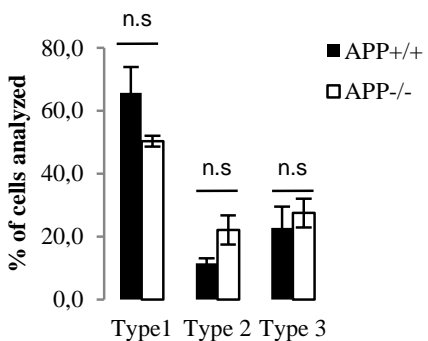

Type1 Type 2 Type 3

B
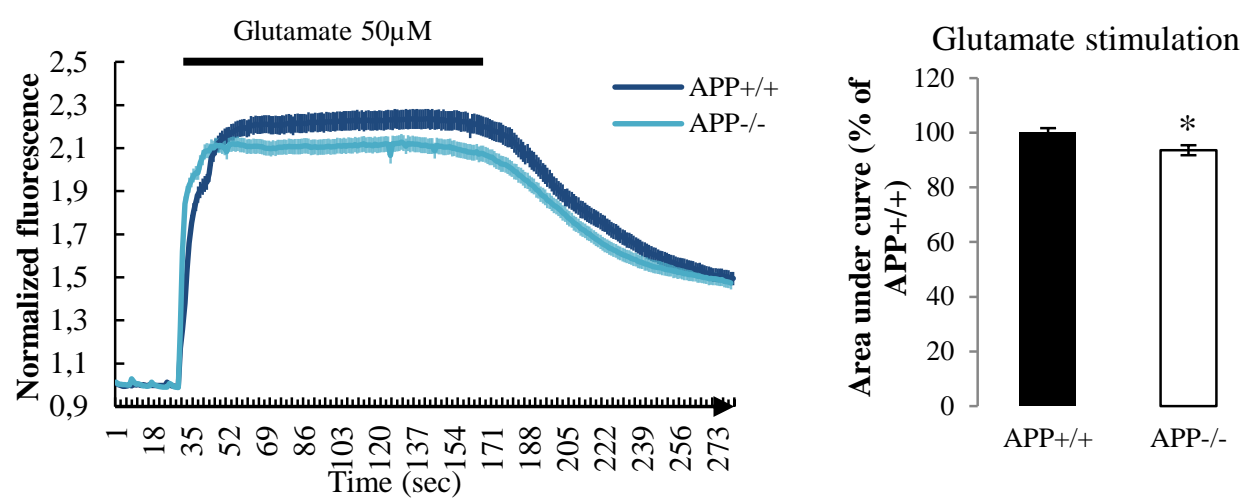

Figure S6: Modification in glutamate responses in APP-/- neurons measured by intracellular calcium imaging. Neuronal activity was measured at DIV7 by calcium imaging. A) Left panel. Different calcium responses were observed after stimulation with $50 \mu \mathrm{M}$ glutamate and classified as described by Prickering and co-workers (Prickering et al. 2008) between neuronal and non-neuronal responses. To note $\mathrm{X}$-axe graduation correspond to 20 sec. Right panel. The proportion of cells displaying Type 1, 2 or 3 response was quantified in three independent experiments $(n=9, N=3)$. n.s.= non-significant. Student-t test. B) Normalized fluorescence trace (mean \pm SEM) measured in APP+/+ and APP-/- neurons upon perfusion for $150 \mathrm{sec}$ with $50 \mu \mathrm{M}$ glutamate. The area under curve (AUC) was quantified for 50 neurons per coverslips. A total of 9 coverslips for each genotype was recorded in three independent experiments $(\mathrm{N}=3)$. The graph on the right shows AUC expressed as percentage of control (APP $+/+)$. $* \mathrm{p}=0,0106$, Student's t-test. 


\section{Supplemental Figure 6: GAD65 positive cells in neuronal cultures}

A

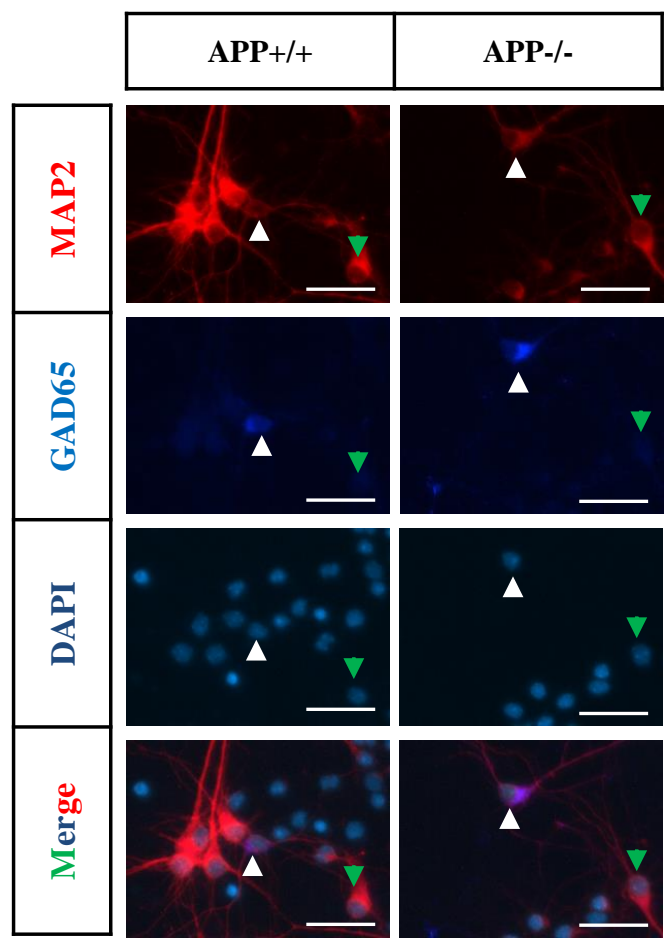

B

GAD65 positive neurons

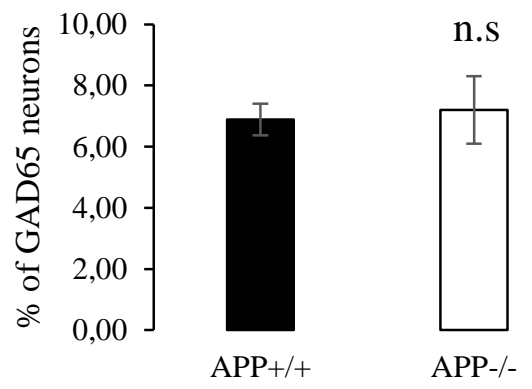

\section{Figure S6: GAD65 positive neurons in primary cortical culture.}

A) Primary culture of cortical neurons after at DIV7. Cultures were immunostained with the neuron specific protein MAP2 (red), GAD65 (dark blue) and DAPI (light blue). Representative 20x micrographs show GAD65 positive neurons (white arrowhead) and GAD65 negative neuron (green arrowhead). B) Images (20x objective) were quantified (10 fields per coverslip for each genotype) in three independent cultures ( $n=30, N=3)$. Results (mean \pm s.e.m) are expressed as percentage of GAD65+ MAP2+ cells (GAD65+ neurons) among all MAP2+ cells (neurons). $\mathrm{n} . \mathrm{s}=$ non-significant, Mann-Whitney test. Scale bar $=20 \mu \mathrm{m}$. 